\title{
An immersed boundary-lattice Boltzmann method for thermal and thermo-solutal problems of Newtonian and non-Newtonian fluids
}

\author{
Cite as: Phys. Fluids 32, 073103 (2020); doi: 10.1063/5.0013977 \\ Submitted: 16 May 2020 - Accepted: 22 June 2020 • \\ Published Online: 8 July 2020
}

Gholamreza Kefayati

AFFILIATIONS
School of Engineering, University of Tasmania, Hobart 7001, Tasmania, Australia

a) Author to whom correspondence should be addressed: gholamreza.kefayati@utas.edu.au

\begin{abstract}
An immersed boundary-lattice Boltzmann method is introduced that can be employed for different thermal and thermo-solutal problems of Newtonian and non-Newtonian fluids. The general macroscopic and mesoscopic equations are presented and discussed. It is shown and proved that the macroscopic equations are satisfied by the proposed lattice Boltzmann equations. This approach removes the limitation of the conventional lattice Boltzmann method in constitutive equations and boundary conditions. To validate the accuracy of the method, it is compared against several cases of complex geometries with curved boundaries for natural convection in enclosures. To demonstrate the ability of this method for the simulation of thermo-solutal flows of non-Newtonian fluids with curved boundaries, double diffusive natural convection of Carreau fluid between a square cylinder and two circular cylinders is investigated and results are reported. Next, double diffusive mixed convection of a Bingham fluid in a cavity with a curved boundary condition is studied.
\end{abstract}

Published under license by AIP Publishing. https://doi.org/10.1063/5.0013977

\section{INTRODUCTION}

Numerical simulation of flow and heat transfer in an enclosure with complex inside geometries and curved boundaries is a challenging topic and has been investigated widely by different approaches.

The immersed boundary method (IBM) is a flexible and strong approach for simulating complex geometries and boundaries. ${ }^{7}$ IBM was introduced in early 1970s for many isothermal and biological systems by Peskin and his colleagues. ${ }^{8-13}$ In this method, elastic boundaries are employed for complex geometries. The deformation in the boundaries is translated in the momentum equation in the form of an external force that has a relation similar to a spring force. It means that the boundaries are known as high stiffness spring that inclines back to its initial position. It should be noted that Lagrangian points are used to study the immersed boundaries, while the mass and momentum equations are solved by using the Eulerian approach. Goldstein et al. ${ }^{14}$ improved the Peskin's method for studying two dimensional flow around cylinders and three dimensional turbulent flow in a channel. Lai and Peskin ${ }^{15}$ adopted a secondorder IBM and compared the obtained results with the first order ones. It was reported that the second order IBM is a better choice for high Reynolds numbers due to the less numerical viscosity. Zhu and Peskin ${ }^{16}$ scrutinized a flexible filament using IBM. They considered the incompressible Navier-Stokes equation with the effects of filament elasticity and air resistance. The delta function approximation, which was just for the fluid velocity, was also examined for the fluid mass density along the immersed filament. Kim and Peskin ${ }^{17}$ explored a penalty immersed boundary method (PIBM) to evaluate the inertial force and the numerical stability. In this approach, two boundaries of the massive elastic boundary and massless boundary were used. Zhu et al. ${ }^{18}$ studied a three dimensional flow past a flexible sheet in a channel by using the Lattice Boltzmann method (LBM) and the IBM for the flexible sheet similar to the technique of Zhu and Peskin. ${ }^{16}$ Tian et al. ${ }^{19}$ modified the PIBM for the flowstructure interaction by using the method of Kim and Peskin. ${ }^{17}$ They tested their work with the results of an elastic filament flapping and the entrainment zone around a cylinder. Zhu et al. ${ }^{20}$ developed their previous IB-LBM code, which was presented by Zhu et al. ${ }^{18}$ from Newtonian fluid to power-law fluid and employed for modeling a three dimensional flexible plate and handling the fluid-plate interaction. 
The cited methods of IBM have been conducted in many recent research works into isothermal flows. ${ }^{21-30}$

The IBM has been developing into various thermal problems using different numerical methods for the past two decades. Pacheco et al. ${ }^{31}$ implemented the IBM for thermal problems over or inside complex geometries. They solved the governing equations in different types of boundary conditions by using the finite volume method (FVM). Zhang et al. ${ }^{32}$ described an IBM for modeling flow over a stationary heated circular cylinder and compared their results with previous numerical and experimental results. Kim et al. ${ }^{33}$ simulated natural convection between a cold cavity and a hot inner circular cylinder using the IBM based on FVM. Wang et al. ${ }^{34}$ extended the IBM with finite difference method (FDM) to solve the heat transfer process. The method was assessed by two different examples of natural convection between concentric cylinders and flow past a stationary circular cylinder. Jeong et al. ${ }^{35}$ proposed an innovative IB-LBM to analyze heat transfer around bluff bodies. They considered natural convection in a cavity with different Rayleigh numbers to confirm the accuracy of the method. Ren et al. ${ }^{36}$ utilized IBM for free and forced convection in different geometries and thermal boundary conditions. They validated their results with previous studies and presented two ways of calculating the average Nusselt number. Liao and $\operatorname{Lin}^{37}$ simulated natural and forced convection in complex geometries at low Reynolds numbers by using the IBM. They examined the method by studying natural convection in an annulus and three dimensional simulation of a heated sphere. Natural and mixed convection of stationary and rotating elliptic cylinders in a square cavity employing IBM were investigated by Liao and Lin. ${ }^{38}$ Ren et al. ${ }^{39}$ proposed an IBM with velocity and temperature corrections. The velocity and temperature corrections were performed by introducing a forcing term in the momentum equation and a heat source term in the energy equation to consider the effect of the IBM. Forced and natural convection problems were examined to validate the method. Ilinca and Hétu ${ }^{40}$ conducted a numerical method using an IBM and a finite element method (FEM) for natural convection between a cold cavity and an inner hot rosette-shaped cylinder. Choi et al. ${ }^{41}$ investigated natural convection in a rhombus enclosure with an inner circular cylinder using IBM. Hu et al. ${ }^{42}$ developed a thermal IB-LBM to study natural convection in a concentric horizontal annulus with a constant heat flux boundary. Wang et al. ${ }^{43}$ proposed an innovative IB-LBM for different thermal boundaries. They validated their results with simulating several benchmarks of natural and mixed convection problems. $\mathrm{Wu}$ et al. ${ }^{44}$ introduced an iterative correction to modify the heat source term in IB-LBM to deal with temperature jump at boundaries. Natural convection between two concentric circular cylinders was simulated to assess the accuracy of the method. Park et al. ${ }^{45}$ simulated fluid-flexible body interactions with heat transfer by IBM. A force term was added to the momentum equation to cover the momentum transfer between the flexible body and the surrounding fluid. Moreover, a heat source was inserted in the energy equation to satisfy the thermal boundary conditions on the curved body. Lee et $a l .{ }^{46}$ adopted PIBM to investigate the fluid-structure-thermal interaction between the surrounding fluid and the flexible flags numerically. Delouei et al. ${ }^{47}$ simulated power-law fluid flow over a heated circular cylinder by IB-LBM. They investigated the effects of power-law index and Reynolds number on fluid flow and heat transfer.
The IBM has been considered for limited isothermal-solutal studies, but it is not utilized in thermo-solutal cases. Lee et al. ${ }^{48}$ introduced an IBM for fluid-solute-structure interaction. The fluid flow part was solved by using incompressible Stokes equations with an external force due to IBM. The chemical species were governed by the advection-electrodiffusion equations. Gong et al. ${ }^{49}$ analyzed the convection-diffusion of mass transfer in permeable moving interfaces. They selected the mass flux as an independent variable. The method evaluated the oxygen transfer in a capillary vessel.

LBM has been known as a significant approach in computational fluid dynamics (CFD) and an effective alternative method for Navier-Stokes and Cauchy equations. ${ }^{50-57}$ Hence, this method has been applied for various problems of fluid flows and heat transfer. ${ }^{58-60}$ In common LBM that the Lattice Boltzmann Equation (LBE) is discretized by the Bhatnagar-Gross-Krook (BGK) approximation, the pressure is proportional to the density and the viscosity is dependent on the collision relaxation time. An LBM was proposed by $\mathrm{Fu}$ et al. ${ }^{61,62}$ that removed the mentioned restrictions on the viscosity and provided the opportunity to select any desirable constitutive equations. Huilgol and Kefayati ${ }^{63,64}$ extended the method in different coordinates and examined the approach for multifarious non-Newtonian fluids. Recently, Kefayati et al. ${ }^{65}$ improved the method for fluid-structure interaction through an isothermal viscoplastic fluid. The fluid-structure interaction in the presence of viscoplastic fluids for rigid and elastic cases was analyzed.

The main aim of this research is to propose a new IB-LBM in order to simulate thermal and solutal flows of Newtonian and nonNewtonian fluids. This method covers all parameters in momentum, energy, and concentration equations. In Sec. II, general macroscopic equations (mass, momentum, energy, and concentration equations) in the presence of the IBM are shown and discussed. In Sec. III, a mesoscopic method based on IB-LBM that satisfies the macroscopic equations is presented. The derivation and detailed steps are indicated in the Appendices. In Sec. IV, the proposed method is validated by previous studies. Then, the introduced method is evaluated by two different non-Newtonian thermo-solutal flows. In the first example, double diffusive natural convection of Carreau fluid between two cold cylinders and a hot square enclosure is simulated. In the second case, double diffusive mixed convection of Bingham fluid is investigated in a lid-driven cavity with a curved boundary.

\section{THE MATHEMATICAL MODEL OF MACROSCOPIC EQUATIONS}

The general macroscopic mass and momentum equations of incompressible fluid flows and conservative materials can be written as

$$
\begin{gathered}
\nabla \cdot \mathbf{u}=0, \\
\rho \frac{d \mathbf{u}}{d t}=\nabla \cdot \boldsymbol{\sigma}+\boldsymbol{\beta}, \quad \boldsymbol{\beta}=\rho \mathbf{b}+\mathbf{f} \cdot,
\end{gathered}
$$

where $t, \mathbf{u}, \rho$, and $\mathbf{b}$ are time, velocity, density, and body force (a force per unit mass), respectively. The total stress tensor is $\boldsymbol{\sigma}=-p \mathbf{1}$ $+\tau$, where $p$ is the pressure and $\tau$ is the extra stress tensor. $\mathbf{f}$ is the force term due to IBM and is expressed as ${ }^{15}$ 


$$
\begin{gathered}
\mathbf{f}(\mathbf{x}, t)=\int \mathbf{F}(s, t) \delta(\mathbf{x}-\mathbf{X}(s, t)) d s, \\
\frac{\partial \mathbf{X}(s, t)}{\partial t}=\mathbf{u}(\mathbf{X}(s, t), t)=\int \mathbf{u}(\mathbf{x}, t) \delta(\mathbf{x}-\mathbf{X}(s, t)) d \mathbf{x}, \\
\mathbf{F}(s, t)=\kappa\left(\mathbf{X}^{e}(s)-\mathbf{X}(s, t)\right) \cdot
\end{gathered}
$$

This force term makes the boundary points to be close to the body surface. $\kappa$ is a positive constant such that $\kappa \gg 1$. Actually, it connects the boundary points $\mathbf{X}$ to fixed equilibrium points $\mathbf{X}^{e}$ with a very stiff spring whose stiffness constant is $\kappa$. The IBM is a mixed Eulerian-Lagrangian FDM for computing the flow interacting with an immersed boundary. The independent Eulerian variables are the Cartesian coordinates $\mathbf{x}$ and the time $t$. The independent Lagrangian variables are the curvilinear material coordinate $s$ and the time $t$. The dependent Eulerian variables are the velocity $\mathbf{u}(\mathbf{x}, t)$, the pressure $p(\mathbf{x}, t)$, the density $\rho(\mathbf{x}, t)$, and the Eulerian force density $\mathbf{f}(\mathbf{x}, t)$. The dependent Lagrangian variables are the immersed position $\mathbf{X}(s, t)$ and the Lagrangian force density $\mathbf{F}(s, t)$.

The $\delta$ is a two-dimensional Dirac delta function and is defined by

$$
\begin{gathered}
\delta(\mathbf{x})=\delta(x) \delta(y), \\
\delta(x)= \begin{cases}\frac{1}{4 h}\left(1+\cos \left(\frac{\pi x}{2 h}\right)\right), & |x| \leq 2 \mathrm{~h}, \\
0, & |x| \geq 2 \mathrm{~h} .\end{cases}
\end{gathered}
$$

where $h$ is the mesh width and is defined by $h=\Delta x=\Delta y$ for a uniform mesh. $\Delta x$ and $\Delta y$ are mesh spacings in $x$ and $y$ directions, respectively. given by

The energy equation of an incompressible continuous media is

$$
\rho \frac{d e}{d t}=\frac{1}{2} \boldsymbol{\sigma}: \mathbf{A}-\nabla \cdot \mathbf{q}+\rho r+\tilde{q},
$$

where $e$ is the internal energy, $\mathbf{q}$ is the heat flux vector, and $r$ is the external supply. A is the first Rivlin-Ericksen tensor and is obtained by $\mathbf{A}=\nabla \mathbf{u}+(\nabla \mathbf{u})^{T}$. The $\tilde{q}$ is the Eulerian heat source that enforces the thermal boundary conditions on the immersed boundary and is expressed as

$$
\tilde{q}(\mathbf{x}, t)=\int Q(s, t) \delta(\mathbf{x}-\mathbf{X}(s, t)) d s .
$$

The heat source $Q$ is calculated on the Lagrangian grid,

$$
Q(s, t)=\frac{1}{\Delta t}\left(T^{e}(s)-T(s, t)\right),
$$

where $\Delta t$ is the time step. The temperature of the massive boundary denoted by $T^{e}$ is determined by the thermal boundary conditions imposed on the body. $T(s, t)$ is the temperature of the massless boundary obtained from the local fluid temperature,

$$
T(s, t)=\int T(\mathbf{x}, t) \delta(\mathbf{x}-\mathbf{X}(s, t)) d \mathbf{x} \cdot
$$

The general concentration equation in incompressible fluid flow is described as follows:

$$
\frac{\partial C}{\partial t}=-\nabla \cdot \mathbf{j}+R+\tilde{j}
$$

where $C$ is the concentration, $\mathbf{j}$ is the total mass flux vector, and $R$ represents the reaction rate for a homogeneous chemical reaction. $\tilde{j}$ is the mass flux that enforces the solutal boundary conditions on the immersed boundary and is defined as

$$
\tilde{j}(\mathbf{x}, t)=\int J(s, t) \delta(\mathbf{x}-\mathbf{X}(s, t)) d s .
$$

The parameter $J$ on the Lagrangian grid is calculated as

$$
J(s, t)=\frac{1}{\Delta t}\left(C^{e}(s)-C(s, t)\right) .
$$

The concentration of the massive boundary denoted by $C^{e}$ in Eq. (14) is determined by the concentration boundary conditions imposed on the body. $C(s, t)$ is the concentration of the massless boundary and is computed as

$$
C(s, t)=\int C(\mathbf{x}, t) \delta(\mathbf{x}-\mathbf{X}(s, t)) d \mathbf{x} \cdot
$$

\section{THE MATHEMATICAL MODEL OF MESOSCOPIC EQUATIONS}

To satisfy the continuity and momentum Eqs. (1) and (2), a function $f_{i}$ is defined as (see Appendix A)

$$
\frac{\partial f_{i}}{\partial t}+\chi_{i} \cdot \nabla_{\mathbf{x}} f_{i}-B_{i}=-\frac{1}{\epsilon \phi}\left(f_{i}-f_{i}^{e q}\right)
$$

where $\phi$ is the relaxation time and $\chi_{i}$ is the particle velocity. $\epsilon$ is a small parameter to be prescribed when numerical simulations are considered.

In order to obtain the macroscopic energy and concentration equations, the following internal energy distribution function $g_{\alpha}$ and the internal concentration distribution function $h_{i}$ are given by (see Appendixes B and C)

$$
\begin{aligned}
& \frac{\partial g_{i}}{\partial t}+\chi_{i} \cdot \nabla_{\mathbf{x}} g_{i}-G_{i}=-\frac{1}{\varepsilon \phi}\left(g_{i}-g_{i}^{e q}\right), \\
& \frac{\partial h_{i}}{\partial t}+\chi_{i} \cdot \nabla_{\mathbf{x}} h_{i}-H_{i}=-\frac{1}{\varepsilon \phi}\left(h_{i}-h_{i}^{e q}\right) .
\end{aligned}
$$

The equilibrium density distribution function $f_{i}^{e q}$ based on the provided information in Appendix A can be written as

$$
f_{i}^{e q}=K_{i}+\chi_{i} \cdot \mathbf{L}_{i}+\left(\chi_{i} \otimes \chi_{i}\right): \mathbf{M}_{i}
$$

where $\mathbf{L}_{i}$ is a vector and $\mathbf{M}_{i}$ is a symmetric matrix. To limit the scope of this study, we just concentrate on two dimensional cases and the flows are assumed to occur in a two dimensional setting underpinned by a D2Q9 lattice. The particle velocity over D2Q9 is given by

$$
\chi_{i}= \begin{cases}(0,0), & i=0 \\ \lambda\left(\cos \Theta_{i}, \sin \Theta_{i}\right), & i=1,3,5,7 \\ \lambda \sqrt{2}\left(\cos \Theta_{i}, \sin \Theta_{i}\right), & i=2,4,6,8,\end{cases}
$$

where $\Theta_{i}$ is defined by $\Theta_{i}=(i-1) \pi / 4, i=1, \ldots, 8$. The constant $\lambda$ has to be chosen with consideration to the numerical stability. The method for finding the parameter $\lambda$ is described in Refs. 63 and 64. 
The relation between the above parameters and macroscopic values is ${ }^{63,64}$

$$
\begin{aligned}
& K_{0}=\rho-\frac{\rho|\mathbf{u}|^{2}}{\lambda^{2}}+\frac{\sigma_{x x}+\sigma_{y y}}{2}, \quad K_{i}=0, i=1,2, \ldots, 8, \\
& \mathbf{L}_{1}=\frac{\rho \mathbf{u}}{2 \lambda^{2}}=\mathbf{L}_{i}, i=1,3,5,7 ; \quad \mathbf{L}_{i}=\mathbf{0}, i=0,2,4,6,8 .
\end{aligned}
$$

For the matrices $\mathbf{M}_{i}, \mathbf{M}_{0}=0, \mathbf{M}_{1}=\mathbf{M}_{i}, i=1,3,5,7, \mathbf{M}_{2}=\mathbf{M}_{i}, i=2,4$, 6,8 , where

$$
\begin{gathered}
\mathbf{M}_{1}=\left[\begin{array}{cc}
M_{11} & 0 \\
0 & M_{22}
\end{array}\right], \quad M_{11}=\frac{1}{2 \lambda^{4}}\left(\rho u^{2}-\sigma_{x x}\right), \\
M_{22}=\frac{1}{2 \lambda^{4}}\left(\rho v^{2}-\sigma_{y y}\right), \\
\mathbf{M}_{2}=\left[\begin{array}{cc}
0 & M_{12} \\
M_{21} & 0
\end{array}\right], \quad M_{12}=M_{21}=\frac{1}{8 \lambda^{4}}\left(\rho u v-\sigma_{x y}\right) .
\end{gathered}
$$

The parameter of $B_{i}$ can cover all force terms. It is the combination of the force term due to the immersed boundary and other external forces acting on the fluid, e.g., the gravity. The body force term $B_{i}$ is evaluated by

$$
\begin{gathered}
B_{i}=0, \quad i=0,2,4,6,8, \\
B_{i}=\frac{\beta}{2 \lambda^{2}} \cdot \chi_{i}, \quad i=1,3,5,7 .
\end{gathered}
$$

The equilibrium energy distribution function $g_{i}^{e q}$ is defined as

$$
g_{i}^{e q}=I_{i}+\chi_{i} \cdot \mathbf{E}_{i}
$$

where the scalars $I_{i}$ are such that $I_{i}=I_{1}, \alpha=1,3,5,7$, and $I_{i}=I_{2}$, $i=2,4,6,8$, and set

$$
I_{0}=\rho e_{t}, \quad I_{1}=I_{2}=0 .
$$

$e_{t}$ is the total energy given by the sum of the internal $(e)$ and kinetic energies, i.e.,

$$
e_{t}=e+\frac{1}{2}|\mathbf{u}|^{2}
$$

The vectors $\mathbf{E}_{i}$ are defined as

$$
\begin{gathered}
\mathbf{E}_{0}=\mathbf{0}, \mathbf{E}_{i}=\mathbf{E}_{1}, i=1,3,5,7 ; \mathbf{E}_{i}=\mathbf{E}_{2}, i=2,4,6,8, \\
\mathbf{E}_{1}=\frac{1}{2 \lambda^{2}}\left[\left(\rho e_{t}-\boldsymbol{\sigma}\right) \mathbf{u}+\mathbf{q}\right], \quad \mathbf{E}_{2}=\mathbf{0} .
\end{gathered}
$$

The parameter $G_{i}$ refers to the external supply, e.g., radiation in the energy equation, and can be expressed as

$$
\begin{gathered}
G_{i}=\frac{1}{2 \lambda^{2}} \boldsymbol{\beta} \cdot\left(\boldsymbol{\chi}_{i} \otimes \chi_{i}\right) \mathbf{u}-\frac{1}{4 \lambda^{2}} \operatorname{\rho r}\left(\boldsymbol{\chi}_{i} \otimes \chi_{i}\right): \mathbf{1}-\frac{1}{4 \lambda^{2}} \tilde{q}, \\
i=1,5,7, \\
G_{i}=0, i=0,2,4,6,8,
\end{gathered}
$$

where $\boldsymbol{\beta}\left(\beta_{x}, \beta_{y}\right)$ can be shown as

$$
\begin{aligned}
& \boldsymbol{\beta} \cdot\left(\chi_{1} \otimes \chi_{1}\right) \mathbf{u}=\boldsymbol{\beta} \cdot\left(\chi_{5} \otimes \chi_{5}\right) \mathbf{u}=\lambda^{2} \beta_{x} u, \\
& \boldsymbol{\beta} \cdot\left(\chi_{3} \otimes \chi_{3}\right) \mathbf{u}=\boldsymbol{\beta} \cdot\left(\chi_{7} \otimes \chi_{7}\right) \mathbf{u}=\lambda^{2} \beta_{y} v,
\end{aligned}
$$

$$
\left(\chi_{i} \otimes \chi_{i}\right): \mathbf{1}=\lambda^{2}, \quad i=1,3,5,7 .
$$

The equilibrium concentration distribution function $h_{i}^{e q}$ is found by

$$
h_{i}^{e q}=Z_{i}+\chi_{i} \cdot \mathbf{W}_{i} .
$$

One way of satisfying the above is to assume, as before, that the scalars $Z_{i}$ are such that $Z_{i}=Z_{1}, i=1,3,5,7$, and $Z_{i}=Z_{2}, i=2$, $4,6,8$, and set

$$
Z_{0}=C, \quad Z_{1}=Z_{2}=0 .
$$

In addition, it is assumed that the vectors $\mathbf{W}_{\alpha}$ are defined through $\mathbf{W}_{0}=\mathbf{0}, \mathbf{W}_{i}=\mathbf{W}_{1}, i=1,3,5,7, \mathbf{W}_{i}=\mathbf{W}_{2}, i=2,4,6,8$, where

$$
\mathbf{W}_{1}=\frac{\mathbf{j}}{2 \lambda^{2}}, \quad \mathbf{W}_{2}=\mathbf{0} \text {. }
$$

$H_{i}$ is the external supply in the concentration equation and is calculated as

$$
\begin{gathered}
H_{i}=-\frac{1}{4 \lambda^{2}}(R+\tilde{j}), \quad i=1,3,5,7, \\
H_{i}=0, i=0,2,4,6,8 .
\end{gathered}
$$

$f_{i}, g_{i}$, and $h_{i}$ are divided into two parts of streaming and collision parts using the splitting method. The streaming parts are written as

$$
\begin{gathered}
\frac{\partial f_{i}(\mathbf{x}, t)}{\partial t}+\chi_{i} \cdot \nabla_{\mathbf{x}} f_{i}(\mathbf{x}, t)-B_{i}=0, \\
\frac{\partial g_{i}(\mathbf{x}, t)}{\partial t}+\chi_{i} \cdot \nabla_{\mathbf{x}} g_{i}(\mathbf{x}, t)-G_{i}=0, \\
\frac{\partial h_{i}(\mathbf{x}, t)}{\partial t}+\chi_{i} \cdot \nabla_{\mathbf{x}} h_{i}(\mathbf{x}, t)-H_{i}=0 \cdot .
\end{gathered}
$$

The collision parts are derived as

$$
\begin{gathered}
\frac{\partial f_{i}(\mathbf{x}, t)}{\partial t}=-\frac{1}{\epsilon \phi}\left(f_{i}(\mathbf{x}, t)-f_{i}^{e q}(\mathbf{x}, t)\right), \\
\frac{\partial g_{i}(\mathbf{x}, t)}{\partial t}=-\frac{1}{\epsilon \phi}\left(g_{i}(\mathbf{x}, t)-g_{i}^{e q}(\mathbf{x}, t)\right), \\
\frac{\partial h_{i}(\mathbf{x}, t)}{\partial t}=-\frac{1}{\epsilon \phi}\left(h_{i}(\mathbf{x}, t)-h_{i}^{e q}(\mathbf{x}, t)\right) \cdot .
\end{gathered}
$$

If we set $\epsilon \phi=\Delta t$, the collision parts can be defined as

$$
\begin{aligned}
& f_{i}(\mathbf{x}, t+\Delta t)=f_{i}^{e q}(\mathbf{x}, t), \\
& g_{i}(\mathbf{x}, t+\Delta t)=g_{i}^{e q}(\mathbf{x}, t), \\
& h_{i}(\mathbf{x}, t+\Delta t)=h_{i}^{e q}(\mathbf{x}, t) \cdot,
\end{aligned}
$$

where $f_{i}, g_{i}$, and $h_{i}$ at $t+\Delta t$ are calculated by the obtained values of $f_{i}^{e q}, g_{i}^{e q}$ and $h_{i}^{e q}$ from Eqs. (42a), (42b), and (42c). The initial values of $f_{i}^{e q}, g_{i}^{e q}$ and $h_{i}^{e q}$ are determined by the macroscopic boundary conditions. The corresponding macroscopic quantities are updated by the obtained values of $f_{i}, g_{i}$, and $h_{i}$ in the collision parts. The updated macroscopic values upgrade $f_{i}^{e q}, g_{i}^{e q}$, and $h_{i}^{e q}$. 
The criteria error for continuing the numerical procedure is the difference between the sum of the distribution functions and the constant density (as it is an incompressible study). Hence, the correction of macroscopic parameters, including the pressure and the velocity, continues until a very small difference between the sum of the distribution functions and the density as

$$
\left|\rho-\sum_{i=0}^{8} f_{i}(\mathbf{x}, t)\right|<\Lambda,
$$

where the constant density is taken $\rho=1$ and $\Lambda$ is the stopping criterion for the iteration. It is shown ${ }^{61-64}$ that the stopping criterion is defined by $\Lambda=(\Delta t)^{2}$.

In conventional LBM, specific relations for $f_{\alpha}, g_{\alpha}$, and $h_{\alpha}$ should be applied for various boundary conditions, e.g., on-grid and midgrid bounce back for zero velocity, bounce-back for motion boundaries. However, in this method, boundary conditions can be imposed in a manner similar to macroscopic methods. As a result, different Dirichlet and Neumann boundary conditions can be included in various problems similar to macroscopic methods, and no special equations for $f_{\alpha}, g_{\alpha}$, and $h_{\alpha}$ are required to incorporate the boundary conditions. In fact, the boundary conditions are used as the initial values of $f_{i}^{e q}, g_{i}^{e q}$, and $h_{i}^{e q}$.

\section{NUMERICAL EXAMPLES}

Buoyancy-driven flow in enclosures with differentially heated surfaces is a benchmark problem for testing different numerical techniques. It is a simplified model for a wide variety of practical problems including reactor insulation, cooling of radioactive waste containers, ventilation of rooms, fire prevention, solar energy collection, dispersion of waste heat in estuaries, and crystal growth in liquid metals. Many studies have been conducted to analyze the effects of different isothermal bodies inside enclosures on natural convection phenomena and focused on the diverse body shapes, e.g., circular, square, and triangular cylinders. In these studies, high/low temperature inner bodies are surrounded by an outer low/high temperature enclosure, and the conventional Boussinesq approximation is usually used. With dropping of the hydrostatic terms including the hydrostatic pressure $\left(\nabla p_{s}=\rho \mathbf{g}\right)$, the buoyancy term can be written as $\Delta \rho \mathbf{g}$, where $\Delta \rho$ is the variation of density. Gravity acceleration is defined by $\mathbf{g}=-\mathbf{g e}_{\mathbf{y}}$, where $\mathbf{e}_{\mathbf{y}}$ is the unit vector in the $\mathrm{y}$ direction. Applying the Boussinesq approximation, the density variation due to the small change in temperature is obtained by $\Delta \rho=-\beta_{T}(T$ $-T_{C}$ ). Hence, the body force in Eq. (2) for natural convection is $\mathbf{b}=\left[-\beta_{T}\left(T-T_{C}\right)\right]$ g. $\beta_{T}$ and $T_{C}$ are the thermal expansion coefficient and the reference temperature (which is defined by the cold temperature in this study), respectively. The internal energy is equal to $e=h-\frac{p}{\rho} . h$ is enthalpy and is found by $h=C_{p} T$, where $C_{p}$ is the heat capacity. The heat flux for conservative and isotropic material in Eq. (8) following the Fourier's law of conduction is equal to $q=-k \frac{\partial T}{\partial \mathbf{x}}$, where $k$ is the thermal conductivity. In benchmark studies, the viscous dissipation is neglected in the energy equation, so the parameter $\frac{1}{2} \boldsymbol{\tau}: \mathbf{A}$ is eliminated. In addition, it is assumed that the external supply in Eq. (8) is neglected. Consequently, the governing equations are presented as

$$
\nabla \cdot \mathbf{u}=0
$$

$$
\begin{gathered}
\rho \frac{d \mathbf{u}}{d t}=\nabla \cdot \boldsymbol{\sigma}+\rho \beta_{T}\left(T-T_{C}\right) g \mathbf{e}_{\mathbf{y}}+\mathbf{f} \\
\rho C_{p} \frac{d T}{d t}=k \nabla^{2} T+\tilde{q} \cdot
\end{gathered}
$$

The time derivative of a function can be written by $\frac{d}{d t}=\frac{\partial}{\partial t}$ $+\mathbf{u} \cdot \nabla$. For incompressible Newtonian fluid, the extra stress tensor $(\boldsymbol{\tau})$ is defined by $\boldsymbol{\tau}=\eta \mathbf{A}$. Following the same normalization as by Quere, ${ }^{66}$ the applied non-dimensional macroscopic equations of natural convection are presented here. The buoyancy velocity scale is $U=\left(\frac{\alpha}{L}\right) \mathrm{Ra}^{0.5}$, where the Rayleigh number $(\mathrm{Ra})$ is defined by $\mathrm{Ra}=\frac{\rho \beta_{T} g L^{3} \Delta T}{\eta \alpha}$, where $L, \eta$, and $\alpha$ are the characteristic length, dynamic viscosity, and thermal diffusivity, respectively. In order to proceed to the numerical solution of the system, the following nondimensional variables of $t^{*}=\frac{t U}{L}, \quad \mathbf{x}^{*}=\mathbf{x} / L, \quad \mathbf{u}^{*}=\frac{\mathbf{u}}{U}, p^{*}=\frac{p}{\rho U^{2}}$, $T^{*}=\left(T-T_{C}\right) / \Delta T, \Delta T=T_{H}-T_{C}, \quad \boldsymbol{\tau}^{*}=\frac{\tau L}{\eta U}$ are employed. The non-dimensional macroscopic continuity, momentum and energy equations of incompressible flows in a two-dimensional domain containing an immersed boundary in the absence of the viscous dissipation are

$$
\nabla \cdot \mathbf{u}^{*}=0
$$

$$
\begin{gathered}
\frac{\partial \mathbf{u}^{*}}{\partial t^{*}}+\mathbf{u}^{*} \cdot \nabla \mathbf{u}^{*}=-\nabla p^{*}+\frac{\operatorname{Pr}}{\sqrt{\mathrm{Ra}}} \nabla \cdot \tau^{*}+\operatorname{Pr} T^{*} \mathbf{e}_{\mathbf{y}}+\mathbf{f}^{*}, \\
\frac{\partial T^{*}}{\partial t^{*}}+\mathbf{u}^{*} \cdot \nabla T^{*}=\frac{1}{\sqrt{\mathrm{Ra}}} \nabla^{2} T^{*}+\tilde{q}^{*} \cdot
\end{gathered}
$$

where $\operatorname{Pr}$ is the Prandtl number, which is defined by $\operatorname{Pr}=\frac{\eta}{\rho \alpha}$. To study the rate of heat transfer, the local and surface-averaged Nusselt numbers are calculated as follows:

$$
N u=\left(\frac{\partial T^{*}}{\partial n}\right)_{\text {wall }}, \quad N u_{\text {avg }}=\frac{1}{S} \int_{0}^{S} N u d S,
$$

where $n$ represents the direction normal to the wall and $S$ is the length of the surface.

To satisfy the non-dimensional equations (47)-(49) with the proposed LBM, the non-dimensional macroscopic variables $\left(\mathbf{x}^{*}, \mathbf{u}^{*}\right.$, $\left.T^{*}, p^{*}\right)$ and non-dimensional parameters ( $\mathrm{Ra}$ and $\mathrm{Pr}$ ) should be applied. The non-dimensional total stress tensor in Eqs. (21)-(24) is found by $\boldsymbol{\sigma}^{*}=-p^{*} \mathbf{1}+\frac{\operatorname{Pr}}{\sqrt{\mathrm{Ra}}} \boldsymbol{\tau}^{*}$. The force term in LBM in Eq. (25) is specified by $\boldsymbol{\beta}=\operatorname{Pr} T^{*} \mathbf{e}_{\mathbf{y}}+\mathbf{f}^{*}$. The parameter $I_{0}$ in the equilibrium energy distribution function in Eq. (28) is obtained by $I_{0}=T^{*}$. The parameter $\mathbf{E}_{\mathbf{1}}$ in Eq. $(29)$ is equal to $\mathbf{E}_{\mathbf{1}}=\mathbf{u}^{*} T^{*}-\frac{1}{\sqrt{\mathrm{Ra}}} \frac{\partial T^{*}}{\partial \mathbf{x}^{*}}$ and $G_{i}$ is zero. In this case study, there is no mass transfer, and therefore, the concentration distribution function is zero $\left(h_{i}=0\right)$. It should be noted that the time step in all studied cases in this investigation is fixed at $\Delta t=0.0001$.

In the first example, natural convection between a hot inside circular cylinder $\left(T^{*}=1\right)$ and a cold outer square cylinder $\left(T^{*}=0\right)$, which are concentric, is studied. The square cylinder sides are $L$ and the circle radius is $R=0.2 L$. The isotherms and streamlines are validated by the study of Zhang et al. ${ }^{67}$ in Fig. 1 for various Rayleigh numbers of $\mathrm{Ra}=10^{4}$ and $10^{5}$ at $\operatorname{Pr}=0.71$. The calculated average 
(a)
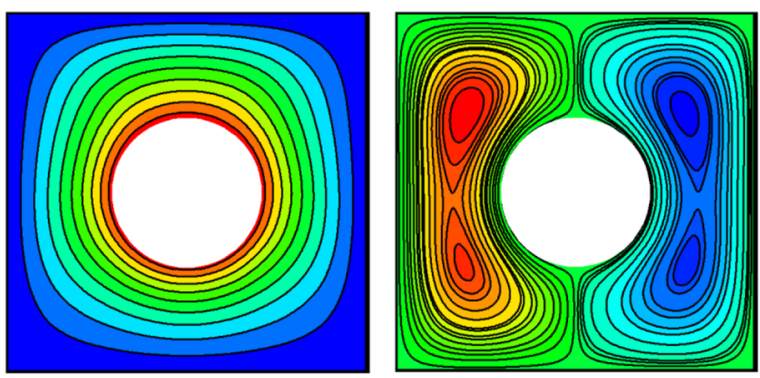

(b)
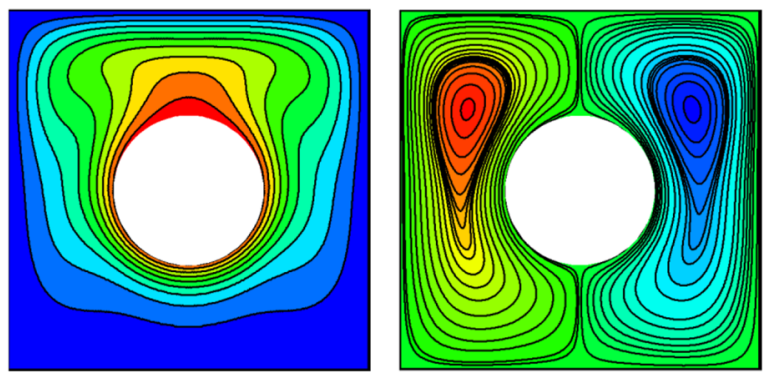

FIG. 1. The isotherms (left column) and streamlines (right column) in Rayleigh numbers of (a) $\mathrm{Ra}=10^{4}$ and (b) $\mathrm{Ra}=10^{5}$ for $\mathrm{Pr}=0.71$.

Nusselt number is compared against previous studies of Zhang et al. ${ }^{67}$ Kim et al. ${ }^{33}$ and Park et al. ${ }^{68}$ in Table I. In the next case, instead of the circular cylinder, an elliptical hot cylinder $\left(T^{*}=1\right)$ is placed at the center of the cold square cylinder $\left(T^{*}=0\right)$, while the horizontal $\left(R_{h}\right)$ and vertical $\left(R_{v}\right)$ radii are fixed at $R_{h}=0.3 L$ and $R_{v}=0.15 \mathrm{~L}$. The isotherms and streamlines are assessed by the study of Zhang et al..$^{67}$ in Fig. 2 for $\mathrm{Ra}=10^{5}$ and $\mathrm{Pr}=0.71$ and confirmed the accuracy of the contours. For the concentric circular and elliptical cases, the grid size of $\Delta x=\Delta y=\frac{1}{301}$ showed low norm of relative error.

Another studied benchmark is two eccentric hot circular cylinders $\left(T^{*}=1\right)$ that are set in vertical positions with the same radius of $R=0.1 L$ inside of a cold cylinder $\left(T^{*}=0\right)$. The length of the square sidewall is $L$ and centers of the circular cylinders are fixed at $\left(x_{c 1}=0.5 L, y_{c 1}=0.3 L\right)$ and $\left(x_{c 2}=0.5 L, y_{c 2}=0.7 L\right)$. The isotherms and streamlines are validated by the study of Park et al. ${ }^{68}$ in Fig. 3 for $\mathrm{Ra}=10^{5}$ and $\operatorname{Pr}=0.71$ and demonstrated good agreement. The grid independence of the solution was tested, and the grid size of $\Delta x=\Delta y=\frac{1}{351}$ showed appropriate results.

TABLE I. Comparison of the average Nusselt number on the one hot circular cylinder between the present result and previous studies in different Rayleigh numbers.

\begin{tabular}{lcccc}
\hline \hline Ra & Present study & Zhang et al. $^{67}$ & Kim et al. $^{33}$ & Park et al. \\
\hline $10^{3}$ & 5.105 & 5.103 & 5.093 & 5.107 \\
$10^{4}$ & 5.116 & 5.087 & 5.108 & 5.128 \\
$10^{5}$ & 7.791 & 7.651 & 7.767 & 7.836 \\
$10^{6}$ & 14.201 & 14.024 & 14.110 & 14.462 \\
\hline \hline
\end{tabular}
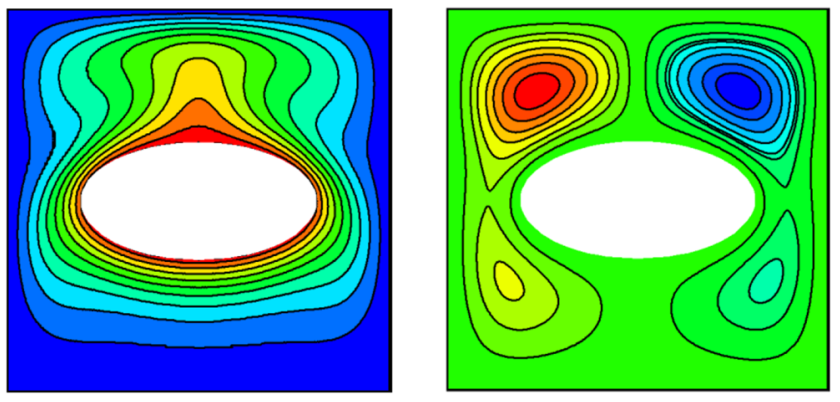

FIG. 2. The isotherms (left column) and streamlines (right column) at $\mathrm{Ra}=10^{5}$ and $\mathrm{Pr}=0.71$.
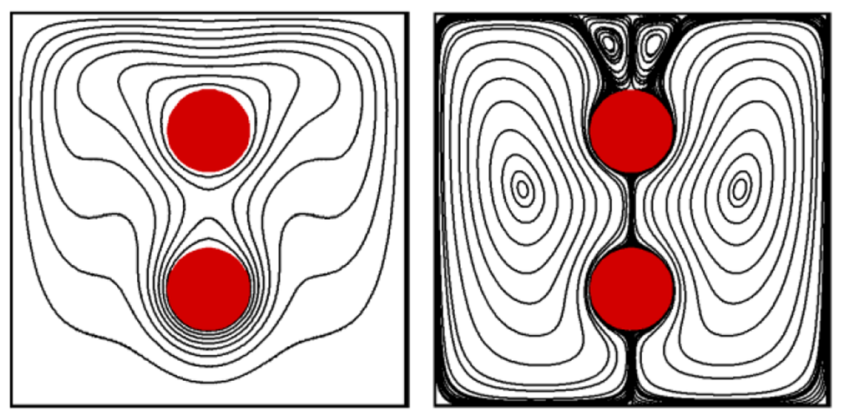

FIG. 3. The isotherms (left column) and streamlines (right column) at $\mathrm{Ra}=10^{5}$ and $\operatorname{Pr}=0.71$.

In the next study, four eccentric hot circular cylinders $\left(T^{*}=1\right)$ inside of a cold square enclosure $\left(T^{*}=0\right)$ in diamond shape with the same radius of $R=0.1 L$ are fixed at $\left(x_{c 1}=0.5 L, y_{c 1}=0.3 L\right)$, $\left(x_{c 2}=0.5 L, y_{c 2}=0.7 L\right),\left(x_{c 3}=0.3 L, y_{c 3}=0.5 L\right)$, and $\left(x_{c 4}=0.7 L, y_{c 4}\right.$ $=0.5 \mathrm{~L})$. The isotherms and streamlines are compared and found to be identical with the study of Mun et al. ${ }^{69}$ in Fig. 4. The grid refinement analysis showed that the grid size of $\Delta x=\Delta y=\frac{1}{401}$ has low norm of relative error.

In the final considered problem of natural convection with curved boundary, a concentric circular annulus is studied and the
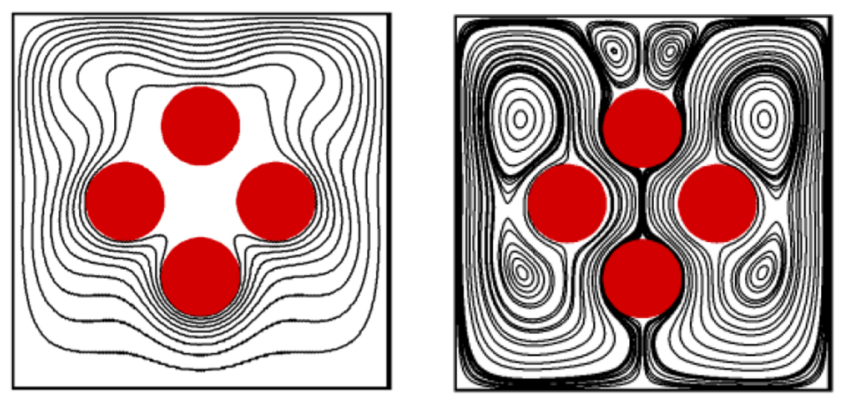

FIG. 4. The isotherms (left column) and streamlines (right column) at $\mathrm{Ra}=10^{5}$ and $\mathrm{Pr}=0.71$. 
(a)
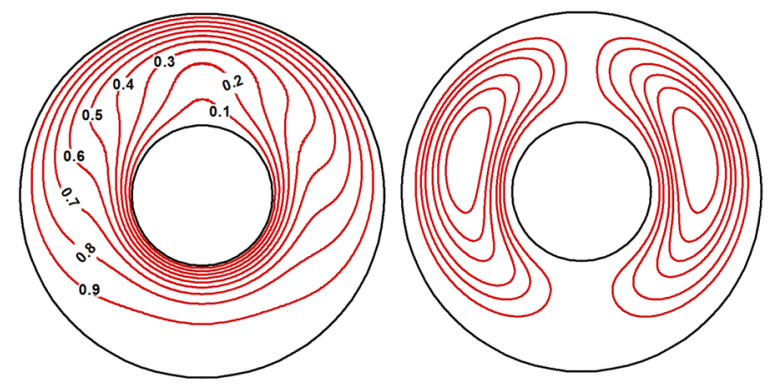

(b)
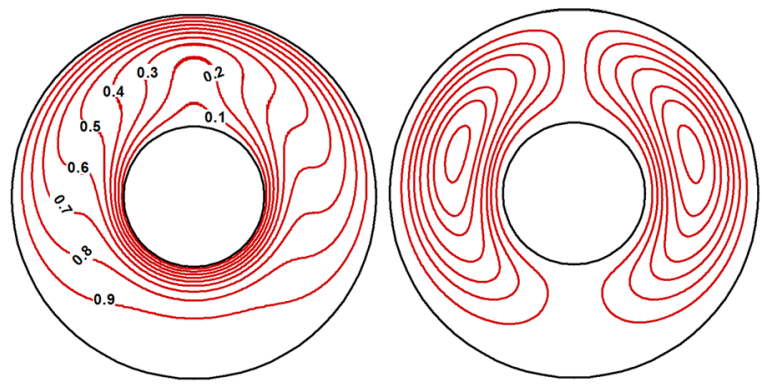

FIG. 5. The isotherms (left column) and streamlines (right column) in Rayleigh numbers of (a) $\mathrm{Ra}=6 \times 10^{3}$ and (b) $\mathrm{Ra}=10^{4}$ for $\mathrm{Pr}=0.71$.

obtained results, including the isotherms, streamlines, and Nusselt number, are compared with previous studies. The temperatures of the inner and outer cylinders with radii of $R_{i}$ and $R_{o}$ are $T_{i}$ and $T_{o}$, respectively. The aspect ratio of the radii $r r$ is defined as $r r=\frac{R_{o}}{R_{i}}$. The applied code for the streamlines and isotherms is validated by the study of Wang et al. ${ }^{70}$ in Fig. 5 for $r r=2.6$ and $\operatorname{Pr}=0.71$.

The heat transfer in this problem can be studied by the equivalent conductivity for the inner cylinder as follows:

$$
\overline{k_{e q i}}=\frac{\ln (r r)}{2 \pi(r r-1)} \int_{0}^{2 \pi}\left(\frac{\partial T}{\partial r}\right) d \theta,
$$

where $r$ and $\theta$ are the radial coordinate and azimuthal coordinate in degrees, respectively. Table II shows a quantitative comparison of the average equivalent heat conductivities of the inner cylinder $\overline{k_{e q i}}$ at various Rayleigh numbers. It is observed that the obtained results agree well with the reference data. Grid independence of the solution was tested for this case, and the grid size of $\Delta x=\Delta y=\frac{1}{451}$ showed low norm of relative error and a good agreement with previous

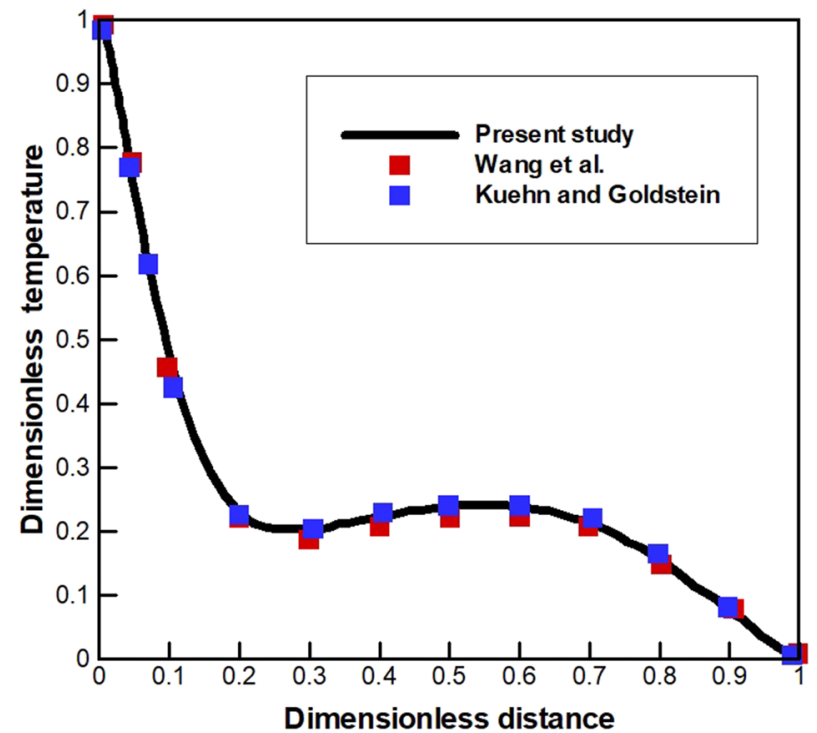

FIG. 6. The comparison of the dimensionless temperature $\left(T-T_{0}\right) /\left(T_{i}-T_{0}\right)$ against the dimensionless distance $\left(R-R_{i}\right) /\left(R_{0}-R_{i}\right)$ between the present study and the experimental results by Kuehn and Goldstein ${ }^{72}$ and the numerical study of Wang et al..$^{34}$ for the azimuthal position of $\theta=120^{\circ}$.

studies that are presented in Table II. In addition, the temperature profile for the azimuthal position of $\theta=120^{\circ}$ has been studied and compared with previous experimental (Kuehn and Goldstein ${ }^{72}$ ) and numerical (Wang et al. ${ }^{34}$ ) investigations. For this purpose, the temperature profile for the azimuthal position of $\theta=120^{\circ}$ has been studied. Figure 6 demonstrates a good agreement between the present result and the mentioned studies.

To demonstrate the ability of this method for studying thermosolutal problems, double diffusive convection as a complex thermosolutal case is studied here. Buoyancy driven due to simultaneous temperature and concentration gradients is known as double diffusive convection. ${ }^{74,75}$ Double diffusive convection is observed in oceanography, astrophysics, geology, and metallurgy widely. By applying the Boussinesq approximation for the same directions of horizontal temperature and concentration gradients and dropping the hydrostatic pressure, the body force term is obtained by $\mathbf{b}=-\left[\beta_{C}\left(C-C_{C}\right)+\beta_{T}\left(T-T_{C}\right)\right] \mathbf{g}$ in Eq. (2). $\beta_{C}$ is the solutal expansion coefficient. Gravity acceleration is defined by $\mathbf{g}=-\mathbf{g e}_{\mathbf{y}}$.

TABLE II. Comparison of average equivalent heat conductivity on the inner cylinder at different Rayleigh numbers.

\begin{tabular}{lccccc}
\hline \hline & Present study & Kuehn and Goldstein & Shu $^{71}$ & Wang et al. & Chen et al. \\
\hline $\mathrm{Ra}=10^{3}$ & 1.079 & 1.081 & 1.082 & 1.076 & 1.091 \\
$\mathrm{Ra}=3 \times 10^{3}$ & 1.391 & 1.404 & 1.397 & 1.381 & 1.393 \\
$\mathrm{Ra}=6 \times 10^{3}$ & 1.713 & 1.736 & 1.715 & 1.695 & 1.698 \\
$\mathrm{Ra}=10^{4}$ & 1.972 & 2.010 & 1.979 & 1.960 & 1.947 \\
$\mathrm{Ra}=5 \times 10^{4}$ & 2.966 & 3.024 & 2.958 & 2.941 & 2.909 \\
\hline \hline
\end{tabular}


The total mass flux $(\mathbf{j})$ is the sum of advective $\left(\mathbf{j}_{a}\right)$, diffusive or dispersive $\left(\mathbf{j}_{d}\right)$, and thermo-diffusive ( $\left.\mathbf{j}_{t}\right)$ mass fluxes in Eq. (12). In double diffusive convection, it is usually assumed that the thermodiffusive or Soret parameter is negligible compared to other formats of mass transfer. The advective mass transfer is obtained by $\mathbf{j}_{a}=\mathbf{u C}$. The diffusive or dispersive mass transfer following the Fick's law can be presented by $\mathbf{j}_{d}=-D \nabla C$, where $D$ is the mass diffusion coefficient. In the presence of mass transfer, there is another heat flux in the energy equation that is diffusive-thermal flux or Dufour parameter. In double diffusion convection, this parameter can be negligible compared to other heat fluxes. Hence, the total heat flux can be written as $q=-k \frac{\partial T}{\partial \mathbf{x}}$. The viscous dissipation also is neglected in the energy equation, so the parameter $\frac{1}{2} \boldsymbol{\tau}: \mathbf{A}$ is removed. Furthermore, the external supply in Eq. (8) is neglected. The dimensional equations with the consideration of the cited assumptions can be written as

$$
\nabla \cdot \mathbf{u}=0
$$

$$
\begin{gathered}
\rho \frac{d \mathbf{u}}{d t}=\nabla \cdot \boldsymbol{\sigma}+\rho\left[\beta_{C}\left(C-C_{C}\right)+\beta_{T}\left(T-T_{C}\right)\right] g \mathbf{e}_{\mathbf{y}}+\mathbf{f}, \\
\rho C_{p} \frac{d T}{d t}=k \nabla^{2} T+\tilde{q}, \\
\frac{d C}{d t}=D \nabla^{2} C+\tilde{j} \cdot .
\end{gathered}
$$

Double diffusive natural convection in eccentric annulus between a square outer cylinder with higher temperature and
Isotherms
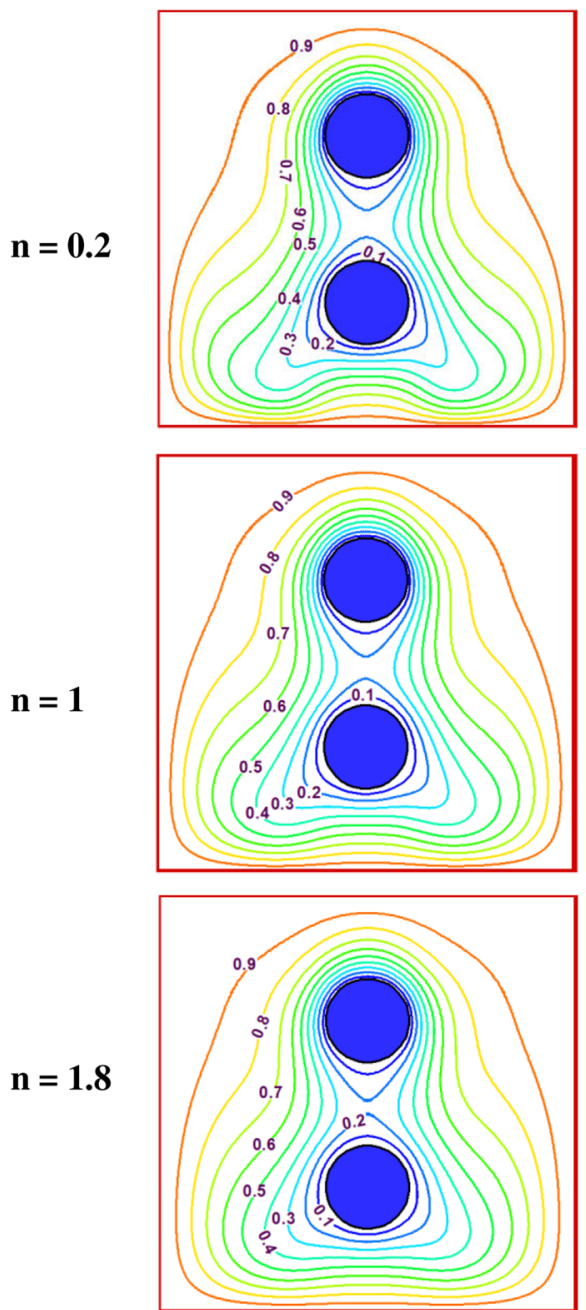

Streamlines
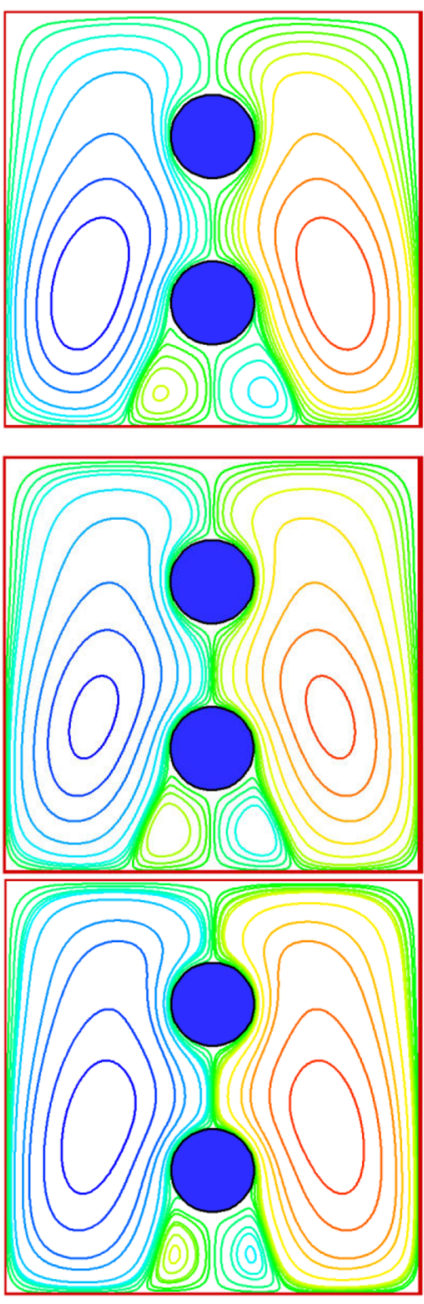

Isoconcentrations
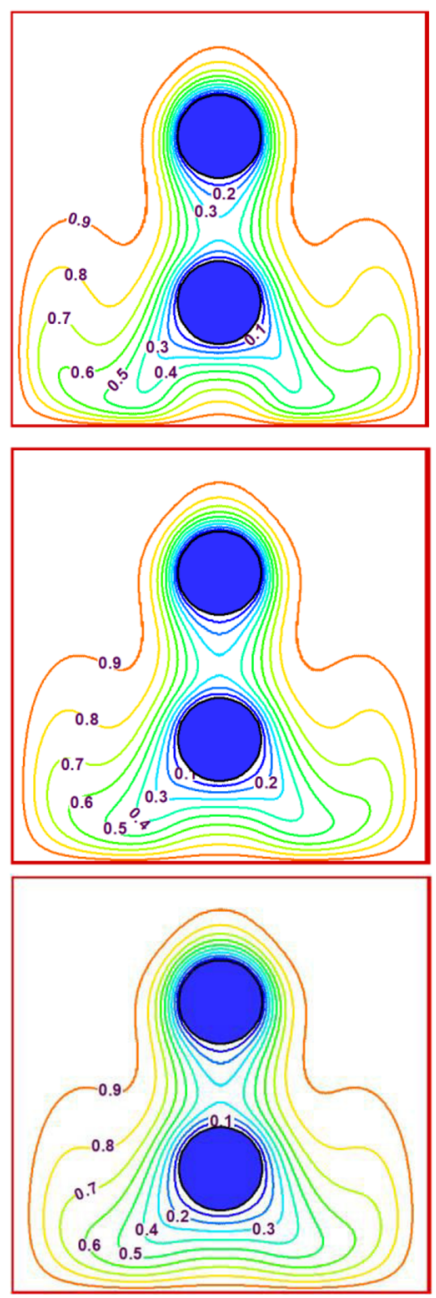

FIG. 7. Comparison of the isotherms, streamlines, and isoconcentrations for different power-law indexes at $\mathrm{Ra}=10^{5}, \mathrm{Pr}=0.1, \mathrm{~N}=0.1, \mathrm{Cu}=1$, and Le $=2.5$ for a Carreau fluid. 
concentration $\left(T^{*}=C^{*}=1\right)$ and two inner circular cylinders with lower temperature and concentration $\left(T^{*}=C^{*}=0\right)$ has been studied for the case of Carreau fluid. The Carreau fluid is a sub-class of nonNewtonian fluid that follows the Carreau model. ${ }^{76}$ This model can be used to analyze the behavior of various chemicals, molten materials, slurries, paints, blood, etc. The extra stress tensor for Carreau fluid is as

$$
\boldsymbol{\tau}=\eta(\dot{\gamma}) \mathbf{A},
$$

where the shear rate $\dot{\gamma}$ is defined as

$$
\dot{\gamma}=\frac{1}{\sqrt{2}} \sqrt{\mathbf{A}: \mathbf{A}} .
$$

The apparent viscosity of Carreau fluid is given by

$$
\eta(\dot{\gamma})=\eta_{\infty}+\left(\eta_{0}-\eta_{\infty}\right)\left[1+(\Gamma \dot{\gamma})^{2}\right]^{(n-1) / 2},
$$

where $\eta_{0}$ and $\eta_{\infty}$ are the viscosities at zero and infinite shear rate. $\eta_{\infty}$ is significantly smaller $\left(10^{3}\right.$ to $10^{4}$ times smaller $)$ than $\eta_{0}$. Hence, $\frac{\eta_{\infty}}{\eta_{0}}$ has been fixed at 0.001 . The non-dimensional variables are similar to the mentioned parameters of the studied natural convection. The non-dimensional concentration is found by $C^{*}=\left(C-C_{C}\right) / \Delta C$, $\Delta C=C_{H}-C_{C}$. The non-dimensional macroscopic equations of diffusive natural convection for incompressible flows in a twodimensional domain containing an immersed boundary in the absence of the viscous dissipation and Soret and Dufour parameters are indicated as

$$
\begin{gathered}
\nabla \cdot \mathbf{u}^{*}=0 \\
\frac{\partial \mathbf{u}^{*}}{\partial t^{*}}+\mathbf{u}^{*} \cdot \nabla \mathbf{u}^{*}=-\nabla p^{*}+\frac{\operatorname{Pr}}{\sqrt{\mathrm{Ra}}} \nabla \cdot \boldsymbol{\tau}^{*}+\operatorname{Pr}\left(T^{*}+\mathrm{N} C^{*}\right) \mathbf{e}_{\mathbf{y}}+\mathbf{f}^{*}, \\
\frac{\partial T^{*}}{\partial t^{*}}+\mathbf{u}^{*} \cdot \nabla T^{*}=\frac{1}{\sqrt{\mathrm{Ra}}} \nabla^{2} T^{*}+\tilde{q}^{*} \\
\frac{\partial C^{*}}{\partial t^{*}}+\mathbf{u}^{*} \cdot \nabla C^{*}=\frac{1}{\operatorname{Le} \sqrt{\mathrm{Ra}}} \nabla^{2} C^{*}+\tilde{j}^{*},
\end{gathered}
$$

where $\mathrm{N}$ and Le are the non-dimensional parameters of buoyancy ratio and Lewis number, respectively, and are defined as

$$
\text { Le }=\frac{\alpha}{D}, \quad \mathrm{~N}=\frac{\Delta C \beta_{C}}{\Delta T \beta_{T}} .
$$

The parameters in LBM are obtained by the non-dimensional macroscopic variables and parameters. The non-dimensional total stress tensor and the force term are defined by $\sigma^{*}=p^{*} 1+\frac{\operatorname{Pr}}{\sqrt{\mathrm{Ra}}} \tau^{*}$ and $\boldsymbol{\beta}=\operatorname{Pr}\left(T^{*}+\mathrm{N} C^{*}\right) \mathbf{e}_{\mathbf{y}}+\mathbf{f}^{*}$. The parameters in the equilibrium energy distribution function are $I_{0}=T^{*}, \mathbf{E}_{\mathbf{1}}=\mathbf{u}^{*} T^{*}-\frac{1}{\sqrt{\mathrm{Ra}}} \frac{\partial T^{*}}{\partial \mathbf{x}^{*}}$, and $G_{i}$ is zero. The parameters in the equilibrium concentration distribution function are $Z_{0}=C^{*}, \mathbf{W}_{\mathbf{1}}=\mathbf{u}^{*} C^{*}-\frac{1}{\mathrm{Le} \sqrt{\mathrm{Ra}}} \frac{\partial C^{*}}{\partial \mathbf{x}^{*}}$, and $H_{i}$ is zero. The local and surface-averaged Sherwood numbers are calculated using the following equations:

$$
S h=\left(\frac{\partial C^{*}}{\partial n}\right)_{\text {wall }}, \quad S h_{\text {avg }}=\frac{1}{S} \int_{0}^{S} S h d S \cdot .
$$

TABLE III. Comparison of the average Nusselt and Sherwood numbers on the hot walls for different power-law indexes at $\mathrm{Ra}=10^{5}, \mathrm{~N}=0.1, \mathrm{Cu}=1$, and $\mathrm{Le}=2.5$.

\begin{tabular}{lccc}
\hline & $\mathrm{n}=0.2$ & $\mathrm{n}=1$ & $\mathrm{n}=1.8$ \\
\hline$N u_{\text {avg }}$ & 9.3232 & 7.9417 & 7.1587 \\
$S h_{\text {avg }}$ & 11.3631 & 8.1273 & 6.4510 \\
\hline \hline
\end{tabular}

The non-dimensional parameter of $\mathrm{Cu}$ (Carreau number) is introduced and is found by $\frac{\Gamma U}{L} . \Gamma, U$, and $L$ are a time constant, the buoyancy velocity scale, and the characteristic length, respectively. The grid size of $\Delta x=\Delta y=\frac{1}{351}$ is applied for this study. Figure 7 displays the isotherms, isoconcentrations, and streamlines for various power-law indexes at $\mathrm{Ra}=10^{5}, \mathrm{Pr}=0.1, \mathrm{~N}=0.1, \mathrm{Cu}$ $=1$, and $\mathrm{Le}=2.5$. It demonstrates that the gradient of temperature on the hot wall declines with the increase in power-law index. In other words, the thermal boundary layer thickness on the side walls decreases. The streamlines also show that the convection process is augmented slightly by the drop of power-law index. In addition, the isoconcentration movement between the hot walls and the cold cylinder enhances as the power-law index decreases from $n=1.8$ to 0.2 . Hence, mass transfer declines considerably with the increase in power-law index. Table III indicates that the increase in the powerlaw index results in dropping of the average Nusselt and Sherwood numbers.

In the second example for non-Newtonian fluids, double diffusive mixed convection in a differentially heated square enclosure filled with a Bingham fluid is studied. The left wall has a higher temperature and concentration $\left(T^{*}=C^{*}=1\right)$, and the right-hand side wall has a curved shape with lower temperature and concentration $\left(T^{*}=C^{*}=0\right)$. The horizontal walls are adiabatic, and the top wall is driven from the left to right at a constant speed $U_{0}$. The geometry of the present problem is shown in Fig. 8. The right wall is curved and shows elliptical behavior (the horizontal and vertical radii are $\mathrm{a}=0.5 \mathrm{~L}$ and $\mathrm{b}=0.2 \mathrm{~L}$, respectively), while other sides have the length of $L$. Bingham fluid is a special sub-class of non-Newtonian

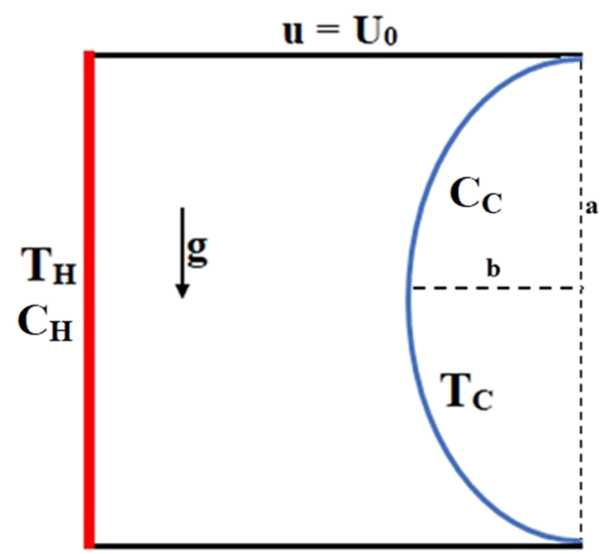

FIG. 8. Geometry of the double-diffusive mixed convection with a curved elliptica cold wall. 
fluids in which the flow field is divided into two sections. In the first section, the fluid is at rest or undergoes a rigid motion, which is called unyielded section. In the second section, the fluid flows behave as a viscous liquid. The constitutive equation for an incompressible Bingham fluid is as

$$
\begin{cases}\mathbf{A}=\mathbf{0}, & |\boldsymbol{\tau}| \leq \tau_{c} \\ \boldsymbol{\tau}=\left(\mu+\frac{\tau_{c}}{\dot{\gamma}}\right) \mathbf{A}, & |\boldsymbol{\tau}|>\tau_{c},\end{cases}
$$

where the Bingham viscosity $\mu$ and the yield stress $\tau_{c}$ are constant. The invariant of $|\boldsymbol{\tau}|$ is defined as

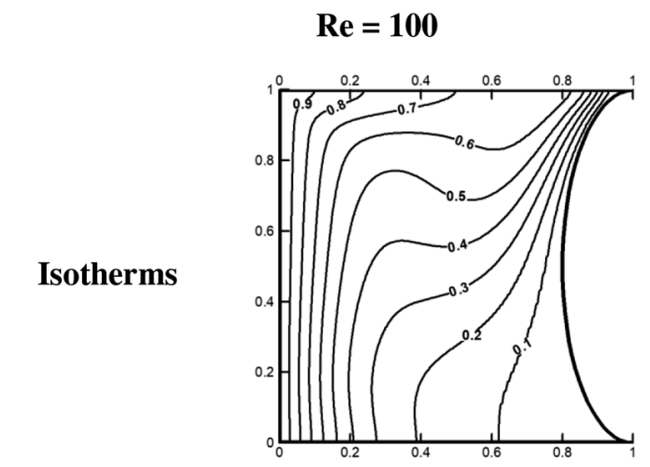

$\mathbf{R e}=\mathbf{5 0 0}$
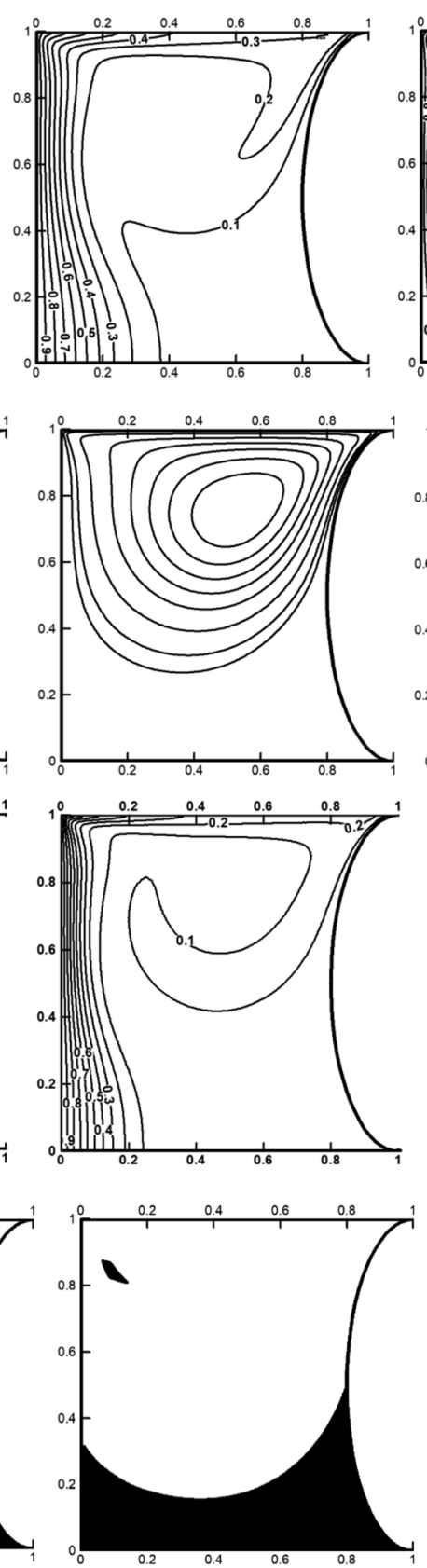

$\operatorname{Re}=1000$
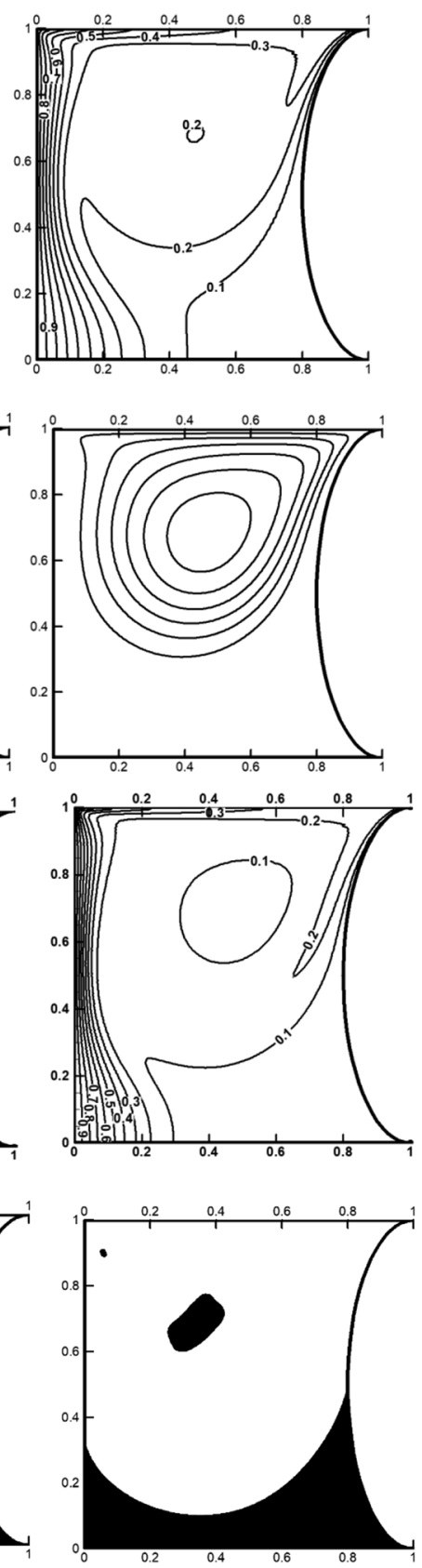

FIG. 9. Comparison of the isotherms, streamlines, isoconcentrations, and yielded/unyielded sections for different Reynolds numbers at $\mathrm{Gr}=10^{4}, \mathrm{~N}=0.1, \mathrm{Pr}=1, \mathrm{Bn}=1$, and Le $=2.5$. 


$$
|\tau|=\sqrt{\frac{1}{2} \tau: \tau} .
$$

Many different approximate models due to the inherent discontinuity of the Bingham model have been introduced and developed by researchers and different software packages. However, in this study, a constitutive equation fully equivalent to the Bingham model is employed as follows: ${ }^{77-79}$

$$
\boldsymbol{\tau}=\mu \mathbf{A}+\sqrt{2} \tau_{c} \boldsymbol{\Lambda}, \quad \mathbf{1}: \mathbf{\Lambda}=0,
$$

where $\Lambda$ is the viscoplasticity constraint tensor where the traceless condition $1: \boldsymbol{\Lambda}=0$ has been imposed on this tensor. The information and conditions of the tensor are discussed in detail in Ref. 7779. The fluid is incompressible and laminar. A two-dimensional domain containing an immersed boundary in the absence of the viscous dissipation and Soret and Dufour parameters is considered. The considered dimensional equations are similar to Eqs. (52)-(55). By applying the cited non-dimensional parameters in the natural convection and applying the $U_{0}$ as the buoyancy velocity scale, the non-dimensional equations are presented as

$$
\nabla \cdot \mathbf{u}^{*}=0
$$

$$
\begin{gathered}
\frac{\partial \mathbf{u}^{*}}{\partial t^{*}}+\mathbf{u}^{*} \cdot \nabla \mathbf{u}^{*}=-\nabla p+\frac{1}{\operatorname{Re}} \nabla \cdot \boldsymbol{\tau}^{*}+\frac{\mathrm{Gr}}{\operatorname{Re} e^{2}}\left(T^{*}+\mathrm{N} C^{*}\right) \mathbf{e}_{\mathbf{y}}+\mathbf{f}^{*} \\
\frac{\partial T^{*}}{\partial t^{*}}+\mathbf{u}^{*} \cdot \nabla T^{*}=\frac{1}{\operatorname{Re} \operatorname{Pr}} \nabla^{2} T^{*}+\tilde{q}^{*} \\
\frac{\partial C^{*}}{\partial t^{*}}+\mathbf{u}^{*} \cdot \nabla C^{*}=\frac{1}{\operatorname{Le} \operatorname{Re} \operatorname{Pr}} \nabla^{2} C^{*}+\tilde{j}^{*}
\end{gathered}
$$

where Grashof (Gr) and Reynolds (Re) numbers are

$$
\mathrm{Gr}=\frac{\rho^{2} \beta_{T} g L^{3}\left(T_{H}-T_{C}\right)}{\mu^{2}}, \quad \operatorname{Re}=\frac{\rho U_{0} L}{\mu},
$$

where the non-dimensional parameter of the Bingham number is written as

$$
\mathrm{Bn}=\frac{\tau_{c} L}{\mu U_{0}}
$$

The non-dimensional extra stress tensor and the force term in LBM are obtained by $\boldsymbol{\sigma}=-p^{*} \mathbf{1}+\frac{1}{\sqrt{\mathrm{Re}}} \boldsymbol{\tau}^{*}$ and $\boldsymbol{\beta}=\frac{\mathrm{Gr}}{\mathrm{Re}^{2}}\left(T^{*}+\mathrm{N} C^{*}\right)+\mathbf{f}^{*}$. The parameters in the equilibrium energy distribution function are $I_{0}=T^{*}, \mathbf{E}_{1}=\mathbf{u}^{*} T^{*}-\frac{1}{\operatorname{Re} \operatorname{Pr}} \frac{\partial T^{*}}{\partial \mathbf{x}^{*}}$, and $G_{i}$ is zero. The parameters in the equilibrium concentration distribution function are $Z_{0}=C^{*}$, $\mathbf{W}_{\mathbf{1}}=\mathbf{u}^{*} C^{*}-\frac{1}{\operatorname{Le} \operatorname{Re} \operatorname{Pr}} \frac{\partial C^{*}}{\partial \mathbf{x}^{*}}$, and $H_{i}$ is zero.

The grid size of $\Delta x=\Delta y=\frac{1}{301}$ is applied for this study. The Grashof and Prandtl numbers are kept at $\mathrm{Gr}=10000$ and $\mathrm{Pr}$ $=1$, respectively. The Reynolds number varies from $\operatorname{Re}=100$ to 500 and 1000. The results of different Reynolds numbers in the forms of isotherms, streamlines, and isoconcentrations are depicted at a Bingham number of $\mathrm{Bn}=1$ in Fig. 9. It is clear that the gradients of isotherms and isoconcentrations on the hot wall augment significantly as the Reynolds number enhances. The average Nusselt and Sherwood numbers in Table IV can prove the pattern as they enhance gradually with the increase in Reynolds number. Also, the
TABLE IV. Comparison of the average Nusselt and Sherwood numbers on the hot walls for different Reynolds numbers at $\mathrm{Gr}=10^{4}, \mathrm{~N}=0.1, \mathrm{Bn}=1$, and $\mathrm{Le}=2.5$.

\begin{tabular}{lccr}
\hline & $\mathrm{Re}=100$ & $\mathrm{Re}=500$ & $\mathrm{Re}=1000$ \\
\hline$N u_{\text {avg }}$ & 3.0929 & 6.2187 & 7.6734 \\
$S h_{\text {avg }}$ & 4.7200 & 9.6938 & 11.7716 \\
\hline \hline
\end{tabular}

yielded (white) and unyielded (black) zones are shown, and the effect of Reynolds number on the size and position of the unyielded zone is demonstrated.

\section{CONCLUSION}

In this work, an IB-LBM has been presented that can effectively recover the macroscopic equations of mass, momentum, energy, and concentration with all parameters. This method provides the opportunity to be applied in various thermal-solutal problems since the LB equations are recovered from general macroscopic equations without any assumptions. In the previous studies into IBM, whether macroscopic or mesoscopic methods, the thermal-solutal problems have not been considered properly and research studies have been focused on isothermal and thermal problems. Although solutal problems are investigated in different areas, they are mostly considered as an isothermal study. Another significant benefit of this approach is the simplicity of this method for studying different models of non-Newtonian fluids and imposing various constitutive equations. In fact, the extra stress tensor can be imposed directly and it is in contrast with common LBMs that viscosity is related to the relaxation time, and this relation can create limitations for studying non-Newtonian fluids. Furthermore, this approach eliminates the complex and time consuming relations from the distribution functions for the boundary condition in conventional LBMs and it makes possible the Neumann and Dirichlet boundary conditions to be implemented by macroscopic parameters straightforwardly.

The introduced method is validated by simulating natural convection in five different cases with curved boundaries. Good agreement between the obtained results and previous studies in these cases was observed, which demonstrates the accuracy of this approach.

It was shown that the present method can be applied effectively for thermo-solutal problems of non-Newtonian fluids where double diffusive natural convection of a Carreau fluid and double diffusive mixed convection of a Bingham fluid were simulated in geometries with curved boundaries.

\section{APPENDIX A: THE DISCRETE PARTICLE DISTRIBUTION FUNCTION}

The following Chapman-Enskog expansion is used:

$$
f_{i}=f_{i}^{e q}+\varepsilon f_{i}^{(1)}+\varepsilon^{2} f_{i}^{(2)}+O\left(\varepsilon^{3}\right) .
$$

The following relations must hold:

$$
\sum_{i=0}^{8} f_{i}^{e q}=\rho,
$$




$$
\begin{gathered}
\sum_{i=0}^{8} f_{i}^{e q} \chi_{i}=\rho \mathbf{u}, \quad \mathbf{u}=u \mathbf{i}+v \mathbf{j}, \\
\sum_{i=0}^{8} f_{i}^{e q}\left(\boldsymbol{\chi}_{i} \otimes \chi_{i}\right)=\mathbf{M}, \\
\sum_{i=0}^{8} f_{i}^{(n)}=0, \quad n \geq 1, \\
\sum_{i=0}^{8} f_{i}^{(n)} \boldsymbol{\chi}_{i}=0, \quad n \geq 1, \\
\sum_{i=0}^{8} B_{i}=0, \\
\sum_{i=0}^{8} B_{i} \boldsymbol{\chi}_{i}=\boldsymbol{\beta} .
\end{gathered}
$$

M has the matrix form,

$$
\mathbf{M}=\left[\begin{array}{ll}
\rho u^{2}-\sigma_{x x} & \rho u v-\sigma_{x y} \\
\rho u v-\sigma_{x y} & \rho v^{2}-\sigma_{y y}
\end{array}\right] .
$$

Substituting the expression for $f_{i}$ in (A1) into Eq. (16), we find that

$$
\frac{\partial f_{i}^{e q}}{\partial t}+\chi_{i} \cdot \nabla_{\mathbf{x}} f_{i}^{e q}-B_{i}=-\frac{1}{\phi} f_{i}^{(1)}+O(\varepsilon)
$$

(A10) can be rewritten as

$$
\frac{\partial f_{i}^{e q}}{\partial t}+\nabla \cdot\left(f_{i}^{e q} \boldsymbol{\chi}_{i}\right)-B_{i}=-\frac{1}{\phi} f_{i}^{(1)}+O(\varepsilon)
$$

Summing the equation above, we obtain

$$
\frac{\partial}{\partial t}\left(\sum_{i=0}^{8} f_{i}^{e q}\right)+\nabla \cdot\left(\sum_{i=0}^{8} f_{i}^{e q} \chi_{i}\right)+\left(\sum_{i=0}^{8} B_{i}\right)=-\frac{1}{\phi} \sum_{i=0}^{8} f_{i}^{(1)}+O(\varepsilon)
$$

Using Eqs. (A2), (A3), and (A7) and recalling that $\rho$ is a constant, the above equation reduces to

$$
\rho(\nabla \cdot \mathbf{u})=0+O(\varepsilon) .
$$

(A13) demonstrates that the continuity equation of an incompressible medium is satisfied.

Multiplying (A10) by $\boldsymbol{\chi}_{i}$,

$$
\frac{\partial f_{i}^{e q} \chi_{i}}{\partial t}+\left(\chi_{i} \cdot \nabla_{\mathrm{x}} f_{i}^{e q}\right) \chi_{i}-B_{i} \chi_{i}=-\frac{1}{\phi} \chi_{i} f_{i}^{(1)}+O(\varepsilon)
$$

We also have

$$
\nabla \cdot\left(f_{i}^{e q} \chi_{i} \otimes \chi_{i}\right)=\left(\xi_{i} \cdot \nabla_{\mathbf{x}} f_{i}^{e q}\right) \chi_{i},
$$

and summing over $i$, one obtains

$$
\begin{gathered}
\frac{\partial}{\partial t}\left(\sum_{i=0}^{8} f_{i}^{e q} \boldsymbol{\chi}_{i}\right)+\nabla \cdot \sum_{i=0}^{8} f_{i}^{e q}\left(\boldsymbol{\chi}_{i} \otimes \boldsymbol{\chi}_{i}\right)-\left(\sum_{i=0}^{8} B_{i} \boldsymbol{\chi}_{i}\right) \\
=-\frac{1}{\phi} \sum_{i=0}^{8} \boldsymbol{\chi}_{i} f_{i}^{(1)}+O(\varepsilon),
\end{gathered}
$$

$$
\rho \frac{\partial \mathbf{u}}{\partial t}+\nabla \cdot \mathbf{M}-\boldsymbol{\beta}=\mathbf{0}+O(\varepsilon) .
$$

Using Eqs. (A1)-(A9), the momentum equation for an incompressible fluid can be found as

$$
\rho \frac{d \mathbf{u}}{d t}-\nabla \cdot \boldsymbol{\sigma}-\boldsymbol{\beta}=\mathbf{0}+O(\varepsilon), \quad \frac{d \mathbf{u}}{d t}=\frac{\partial \mathbf{u}}{\partial t}+(\mathbf{u} \cdot \nabla) \mathbf{u} .
$$

\section{APPENDIX B: INTERNAL ENERGY \\ DISTRIBUTION FUNCTION}

The following relations must hold:

$$
\begin{gathered}
g_{i}=g_{i}^{e q}+\varepsilon g_{i}^{(1)}+\varepsilon^{2} g_{i}^{(2)}+O\left(\varepsilon^{3}\right), \\
\sum_{i=0}^{8} g_{i}^{e q}=\rho e_{t}, \\
\sum_{i=0}^{8} g_{i}^{e q} \boldsymbol{\xi}_{\alpha}=\left(\rho e-\boldsymbol{\sigma}+\frac{1}{2} \rho|\mathbf{u}|^{2}\right) \mathbf{u}+\mathbf{q}, \\
\sum_{i=0}^{8} G_{i}=\rho \boldsymbol{\beta} \cdot \mathbf{u}-\rho r+\tilde{q} .
\end{gathered}
$$

Substituting the expression for $g_{i}$ in (B1) into Eq. (17) and summing over $i$,

$$
\frac{\partial}{\partial t}\left(\sum_{i=0}^{8} g_{i}^{e q}\right)+\nabla \cdot\left(\sum_{i=0}^{8} g_{i}^{e q} \chi_{i}\right)+\left(\sum_{i=0}^{8} G_{i}\right)=-\frac{1}{\phi} \sum_{i=0}^{8} g_{i}^{(1)}+O(\varepsilon) .
$$

Using Eqs. (B2)-(B4), the above equation becomes

$$
\begin{gathered}
\frac{\partial}{\partial t}\left(\rho e_{t}\right)+\nabla \cdot\left(\left(\rho e-\boldsymbol{\sigma}+\frac{1}{2} \rho|\mathbf{u}|^{2}\right) \mathbf{u}+\mathbf{q}\right)+(\rho \boldsymbol{\beta} \cdot \mathbf{u}-\rho r+\tilde{q})=0+O(\varepsilon), \\
e_{t}=e+\frac{1}{2}|\mathbf{u}|^{2}, \\
\nabla \cdot\left(\rho e-\boldsymbol{\sigma}+\frac{1}{2} \rho|\mathbf{u}|^{2}\right) \mathbf{u}=(\rho \nabla e-\nabla \boldsymbol{\sigma}+\rho(\mathbf{u} \cdot \nabla) \mathbf{u}) \cdot \mathbf{u}, \\
\nabla \cdot(\boldsymbol{\sigma u})=(\nabla \cdot \boldsymbol{\sigma}) \cdot \mathbf{u}+\frac{1}{2} \boldsymbol{\sigma}: \mathbf{A} .
\end{gathered}
$$

Combining all the above, we obtain

$$
\rho \frac{d e}{d t}+\left(\rho \frac{d \mathbf{u}}{d t}-\nabla \cdot \boldsymbol{\sigma}-\rho \boldsymbol{\beta}\right) \cdot \mathbf{u}-\frac{1}{2} \boldsymbol{\sigma}: \mathbf{A}+\nabla \cdot \mathbf{q}-\rho r+\tilde{q}=0+O(\varepsilon)
$$

The parameters in the parentheses in Eq. (B10) are the momentum equation, which is equal to zero, and so, it reduces to the general energy equation as follows:

$$
\rho \frac{d e}{d t}-\frac{1}{2} \boldsymbol{\sigma}: \mathbf{A}+\nabla \cdot \mathbf{q}-\rho r+\tilde{q}=0+O(\varepsilon) \cdot .
$$




\section{APPENDIX C: CONCENTRATION DISTRIBUTION FUNCTION}

The following relations must hold:

$$
\begin{gathered}
h_{i}=h_{i}^{e q}+\varepsilon h_{i}^{(1)}+\varepsilon^{2} h_{i}^{(2)}+O\left(\varepsilon^{3}\right), \\
\sum_{i=0}^{8} h_{i}^{e q}=C \\
\sum_{i=0}^{8} h_{i}^{e q} \boldsymbol{\xi}_{\alpha}=\mathbf{j} \\
\sum_{i=0}^{8} H_{i}=R+\tilde{j} .
\end{gathered}
$$

Substituting the expression for $h_{i}$ in (C1) into Eq. (18) and summing over $i$,

$$
\frac{\partial}{\partial t}\left(\sum_{i=0}^{8} h_{i}^{e q}\right)+\nabla \cdot\left(\sum_{i=0}^{8} h_{i}^{e q} \chi_{i}\right)+\left(\sum_{i=0}^{8} H_{i}\right)=-\frac{1}{\phi} \sum_{i=0}^{8} h_{i}^{(1)}+O(\varepsilon) \cdot .
$$

Using Eqs. (C2)-(C4), the above equation becomes

$$
\frac{\partial}{\partial t} C+\nabla \cdot \mathbf{j}+R+\tilde{j}=0+O(\varepsilon) \cdot
$$

\section{DATA AVAILABILITY}

The data that support the findings of this study are available from the corresponding author upon reasonable request.

\section{REFERENCES}

${ }^{1}$ M. Y. Ha, I.-K. Kim, H. S. Yoon, and S. Lee, "Unsteady fluid flow and temperature fields in a horizontal enclosure with an adiabatic body," Phys. Fluids 14, 3189 (2002).

${ }^{2}$ J. R. Lee, M. Y. Ha, S. Balachandar, H. S. Yoon, and S. S. Lee, "Natural convection in a horizontal layer of fluid with a periodic array of square cylinders in the interior," Phys. Fluids 16, 1273 (2004).

${ }^{3}$ Z. Chen, C. Shu, L. M. Yang, X. Zhao, and N. Y. Liu, "Immersed boundarysimplified thermal lattice Boltzmann method for incompressible thermal flows," Phys. Fluids 32, 013605 (2020).

${ }^{4}$ Y. Kim and C. S. Peskin, "A penalty immersed boundary method for a rigid body in fluid," Phys. Fluids 28, 033603 (2016).

${ }^{5}$ C.-C. Liao and C.-A. Lin, "Influence of Prandtl number on the instability of natural convection flows within a square enclosure containing an embedded heated cylinder at moderate Rayleigh number," Phys. Fluids 27, 013603 (2015).

${ }^{6}$ H. S. Yoon, M. Y. Ha, B. S. Kim, and D. H. Yu, "Effect of the position of a circular cylinder in a square enclosure on natural convection at Rayleigh number of $10^{7}$," Phys. Fluids 21, 047101 (2009).

${ }^{7}$ R. Mittal and G. Iaccarino, "Immersed boundary methods," Annu. Rev. Fluid Mech. 37, 239 (2005).

${ }^{8}$ C. S. Peskin, "Flow patterns around heart valves: A numerical method," J. Comput. Phys. 10, 252 (1972).

${ }^{9}$ C. S. Peskin, "Numerical analysis of blood flow in the heart," J. Comput. Phys. 25, 220 (1977).

${ }^{10}$ M. F. McCracken and C. S. Peskin, "A vortex method for blood flow through heart valves,” J. Comput. Phys. 35, 183 (1980).
${ }^{11}$ C. S. Peskin and D. M. McQueen, "A three-dimensional computational method for blood flow in the heart I. Immersed elastic fibers in a viscous incompressible fluid,” J. Comput. Phys. 81, 372 (1989).

${ }^{12}$ C. S. Peskin and D. M. McQueen, "A three-dimensional computational method for blood flow in the heart II. Contractile fibers," J. Comput. Phys. 82, 289 (1989).

${ }^{13}$ C. S. Peskin and B. F. Printz, "Improved volume conservation in the computation of flows with immersed elastic boundaries," J. Comput. Phys. 105, 33 (1989).

${ }^{14}$ D. Goldstein, R. Handler, and L. Sirovich, "Modeling a no-slip flow boundary with an external force field," J. Comput. Phys. 105, 345 (1993).

${ }^{15}$ M.-C. Lai and C. S. Peskin, "An immersed boundary method with formal second-order accuracy and reduced numerical viscosity," J. Comput. Phys. 160, 705 (2000).

${ }^{16}$ L. Zhu and C. S. Peskin, "Simulation of a flapping flexible filament in a flowing soap film by the immersed boundary method," J. Comput. Phys. 179, 452 (2002).

${ }^{17}$ Y. Kim and C. S. Peskin, "Penalty immersed boundary method for an elastic boundary with mass," Phys. Fluids 19, 053103 (2007).

${ }^{18}$ L. Zhu, G. He, S. Wang, L. Miller, X. Zhang, Q. You, and S. Fang, “An immersed boundary method based on the lattice Boltzmann approach in three dimensions, with application," Comput. Appl. Math. 61, 3506 (2011).

${ }^{19}$ F.-B. Tian, H. Luo, L. Zhu, J. C. Liao, and X.-Y. Lu, “An efficient immersed boundary-lattice Boltzmann method for the hydrodynamic interaction of elastic filaments," J. Comput. Phys. 230, 7266 (2011).

${ }^{20}$ L. Zhu, X. Yu, N. Liu, Y. Cheng, and X. Lu, "A deformable plate interacting with a non-Newtonian fluid in three dimensions," Phys. Fluids 29, 083101 (2017).

${ }^{21}$ W. Tong, Y. Yang, and S. Wang, "Characterizing three-dimensional features of vortex surfaces in the flow past a finite plate," Phys. Fluids 32, 011903 (2020).

${ }^{22}$ N. Tofany, Y. M. Low, C.-H. Lee, and Y.-M. Chiew, "Two-phase flow simulation of scour beneath a vibrating pipeline during the tunnel erosion stage," Phys. Fluids 31, 113302 (2019).

${ }^{23}$ J. Ryu, J. Yang, S. G. Park, and H. J. Sung, "Phase-mediated locomotion of two self-propelled flexible plates in a tandem arrangement," Phys. Fluids 32, 041901 (2020).

${ }^{24}$ D. Zhang, G. Pan, L. Chao, and Y. Zhang, "Effects of Reynolds number and thickness on an undulatory self-propelled foil," Phys. Fluids 30, 071902 (2018).

${ }^{25}$ J. W. Park, J. Ryu, and H. J. Sung, "Effects of the shape of an inverted flag on its flapping dynamics," Phys. Fluids 31, 021904 (2019).

${ }^{26}$ R. Subburaj, P. Khandelwal, and S. Vengadesan, "Numerical study of flow past an elliptic cylinder near a free surface,” Phys. Fluids 30, 103603 (2018).

${ }^{27}$ J. Yuan, A. A. Mishra, G. Brereton, G. Iaccarino, and M. Vartdal, "Single-point structure tensors in turbulent channel flows with smooth and wavy walls," Phys. Fluids 31, 125115 (2019).

${ }^{28}$ A. S. Bharadwaj and S. Ghosh, "Numerical investigation of lift enhancement in flapping hover flight,” Phys. Fluids 32, 051901 (2020).

${ }^{29}$ S. Wang, J. Ryu, G. He, F. Qin, and H. J. Sung, "A self-propelled flexible plate with a Navier slip surface," Phys. Fluids 32, 021906 (2020).

${ }^{30}$ F. Liu, G. Liu, and C. Shu, "Fluid-structure interaction simulation based on immersed boundary-lattice Boltzmann flux solver and absolute nodal coordinate formula," Phys. Fluids 32, 047109 (2020).

${ }^{31}$ J. R. Pacheco, A. Pacheco-Vega, T. Rodić, and R. E. Peck, "Numerical simulations of heat transfer and fluid flow problems using an immersed-boundary finite-volume method on non-staggered grids," Numer. Heat Transfer, Part B 48, 1 (2005).

${ }^{32}$ N. Zhang, Z. C. Zheng, and S. Eckels, "Study of heat-transfer on the surface of a circular cylinder in flow using an immersed-boundary method," Int. J. Heat Fluid Flow 29, 1558 (2008).

${ }^{33}$ B. S. Kim, D. S. Lee, M. Y. Ha, and H. S. Yoon, "A numerical study of natural convection in a square enclosure with a circular cylinder at different vertical locations," Int. J. Heat Mass Transfer 51, 1888 (2008).

${ }^{34}$ Z. Wang, J. Fan, K. Luo, and K. Cen, "Immersed boundary method for the simulation of flows with heat transfer," Int. J. Heat Mass Transfer 52, 4510 (2009). 
${ }^{35}$ H. K. Jeong, H. S. Yoon, M. Y. Ha, and M. Tsutahara, "An immersed boundarythermal lattice Boltzmann method using an equilibrium internal energy density approach for the simulation of flows with heat transfer," J. Comput. Phys. 229, 2526 (2010).

${ }^{36}$ W. W. Ren, C. Shu, J. Wu, and W. M. Yang, "Boundary condition-enforced immersed boundary method for thermal flow problems with Dirichlet temperature condition and its applications," Fluids 57, 40 (2012).

${ }^{37}$ C.-C. Liao and C.-A. Lin, "Simulations of natural and forced convection flows with moving embedded object using immersed boundary method," Comput. Methods Appl. Mech. Eng. 213-216, 58 (2012).

${ }^{38}$ C.-C. Liao and C.-A. Lin, "Influences of a confined elliptic cylinder at different aspect ratios and inclinations on the laminar natural and mixed convection flows," Int. J. Heat Mass Transfer 55, 6638 (2012).

${ }^{39}$ W. Ren, C. Shu, and W. Yang, "An efficient immersed boundary method for thermal flow problems with heat flux boundary conditions," Int. J. Heat Mass Transfer 64, 694 (2013).

${ }^{40} \mathrm{~F}$. Ilinca and J.-F. Hétu, "Immersed boundary solution of natural convection in a square cavity with an enclosed rosette-shaped hot cylinder," Numer. Heat Transfer, Part A 65, 1154 (2014).

${ }^{41}$ C. Choi, S. Jeong, M. Y. Ha, and H. S. Yoon, "Effect of a circular cylinder's location on natural convection in a rhombus enclosure," Int. J. Heat Mass Transfer 77, 60 (2014).

${ }^{42}$ Y. Hu, D. Li, S. Shu, and X. Niu, "Study of multiple steady solutions for the 2D natural convection in a concentric horizontal annulus with a constant heat flux wall using immersed boundary-lattice Boltzmann method," Int. J. Heat Mass Transfer 81, 591 (2015).

${ }^{43}$ Y. Wang, C. Shu, and L. M. Yang, "Boundary condition-enforced immersed boundary-lattice Boltzmann flux solver for thermal flows with Neumann boundary conditions," J. Comput. Phys. 306, 237 (2016).

${ }^{44} \mathrm{~J}$. Wu, Y. Cheng, and L. A. Miller, "An iterative source correction based immersed boundary-lattice Boltzmann method for thermal flow simulations," Int. J. Heat Mass Transfer 115, 450 (2017).

${ }^{45}$ S. G. Park, C. B. Chang, B. Kim, and H. J. Sung, "Simulation of fluid-flexible body interaction with heat transfer," Int. J. Heat Mass Transfer 110, 20 (2017).

${ }^{46}$ J. B. Lee, S. G. Park, and H. J. Sung, "Heat transfer enhancement by asymmetrically clamped flexible flags in a channel flow," Int. J. Heat Mass Transfer 116, 1003 (2018).

${ }^{47}$ A. A. Delouei, M. Nazari, M. H. Kayhani, and S. Succi, "Non-Newtonian unconfined flow and heat transfer over a heated cylinder using the direct-forcing immersed boundary-thermal lattice Boltzmann method," Phys. Rev. E 89, 053312 (2014).

${ }^{48}$ P. Lee, B. E. Griffith, and C. S. Peskin, "The immersed boundary method for advection-electrodiffusion with implicit timestepping and local mesh refinement," J. Comput. Phys. 229, 5208 (2010).

${ }^{49} \mathrm{X}$. Gong, Z. Gong, and H. Huang, "An immersed boundary method for mass transfer across permeable moving interfaces," J. Comput. Phys. 278, 148 (2014).

${ }^{50}$ S. Succi, The Lattice Boltzmann Equation: For Fluid Dynamics and Beyond (Oxford University Press, 2001).

${ }^{51} \mathrm{~S}$. Succi, "Mesoscopic modeling of slip motion at fluid-solid interfaces with heterogeneous catalysis," Phys. Rev. Lett. 89, 064502 (2002).

${ }^{52}$ A. A. Mohamad, Lattice Boltzmann Method: Fundamentals and Engineering Applications with Computer Codes (Springer, 2011).

${ }^{53} \mathrm{Z}$. Chen, C. Shu, and D. Tan, "Highly accurate simplified lattice Boltzmann method," Phys. Fluids 30, 103605 (2018).

${ }^{54} \mathrm{Z}$. Chen, C. Shu, and D. Tan, "Three-dimensional simplified and unconditionally stable lattice Boltzmann method for incompressible isothermal and thermal flows," Phys. Fluids 29, 053601 (2017).

${ }^{55} \mathrm{Z}$. Chen, C. Shu, and D. Tan, "Immersed boundary-simplified lattice Boltzmann method for incompressible viscous flows," Phys. Fluids 30, 053601 (2018).

${ }^{56}$ Z. Chen, C. Shu, and L. Q. Zhang, "A simplified axisymmetric lattice Boltzmann method for incompressible swirling and rotating flows," Phys. Fluids 31, 023605 (2019).

${ }^{57}$ H. Z. Yuan, Y. Wang, and C. Shu, "An adaptive mesh refinement-multiphase lattice Boltzmann flux solver for simulation of complex binary fluid flows," Phys. Fluids 29, 123604 (2017).
${ }^{58}$ H. Sajjadi, M. Salmanzadeh, G. Ahmadi, and S. Jafari, "Turbulent indoor airflow simulation using hybrid LES/RANS model utilizing lattice Boltzmann method," Comput. Fluids 150, 66 (2017).

${ }^{59}$ H. Sajjadi, A. A. Delouei, M. Izadi, and R. Mohebbi, "Investigation of MHD natural convection in a porous media by double MRT lattice Boltzmann method utilizing MWCNT- $\mathrm{Fe}_{3} \mathrm{O}_{4} /$ water hybrid nanofluid," Int. J. Heat Mass Transfer 132, 1087 (2019).

${ }^{60}$ H. Sajjadi, A. A. Delouei, M. Atashafrooz, and M. Sheikholeslami, "Double MRT lattice Boltzmann simulation of 3-D MHD natural convection in a cubic cavity with sinusoidal temperature distribution utilizing nanofluid," Int. J. Heat Mass Transfer 126, 489 (2018).

${ }^{61}$ S. C. Fu, R. M. C. So, and W. W. F. Leung, "A discrete flux scheme for aerodynamic and hydrodynamic flows," Comput. Phys. Commun. 9, 1257 (2011). ${ }^{62}$ S. C. Fu, R. M. C. So, and W. W. F. Leung, "Linearized-Boltzmann-typeequation-based finite difference method for thermal incompressible flow," Comput. Fluids 69, 67 (2012).

${ }^{63}$ R. R. Huilgol and G. H. R. Kefayati, "From mesoscopic models to continuum mechanics: Newtonian and non-Newtonian fluids," J. Non-Newtonian Fluid Mech. 233, 146 (2016).

${ }^{64}$ R. R. Huilgol and G. H. R. Kefayati, "A particle distribution function approach to the equations of continuum mechanics in Cartesian, cylindrical and spherical coordinates: Newtonian and non-Newtonian fluids," J. Non-Newtonian Fluid Mech. 251, 119 (2018).

${ }^{65}$ G. H. R. Kefayati, H. Tang, and A. Chan, "Immersed boundary-finite difference lattice Boltzmann method through fluid-structure interaction for viscoplastic fluids," J. Fluid Struct. 83, 238 (2018).

${ }^{66} \mathrm{P}$. L. Quere, "Accurate solutions to the square thermally driven cavity at high Rayleigh number," Comput. Fluids 20, 29 (1991).

${ }^{67} \mathrm{P}$. Zhang, X. Zhang, J. Deng, and L. Song, "A numerical study of natural convection in an inclined square enclosure with an elliptic cylinder using variational multiscale element free Galerkin method," Int. J. Heat Mass Transfer 99, 721 (2016).

${ }^{68}$ Y. G. Park, M. Y. Ha, C. Choi, and J. Park, "Natural convection in a square enclosure with two inner circular cylinders positioned at different vertical locations," Int. J. Heat Mass Transfer 77, 501 (2014).

${ }^{69}$ G. S. Mun, Y. G. Park, H. S. Yoon, M. Kim, and M. Yeong Ha, "Natural convection in a cold enclosure with four hot inner cylinders based on diamond arrays (Part-I: Effect of horizontal and vertical equal distance of inner cylinders)," Int J. Heat Mass Transfer 111, 755 (2017)

${ }^{70} \mathrm{Y}$. Wang, C. Shu, and C. J. Teo, "Thermal lattice Boltzmann flux solver and its application for simulation of incompressible thermal flows," Comput. Fluids 94, 98 (2014).

${ }^{71}$ C. Shu, "Application of differential quadrature method to simulate natural convection in a concentric annulus," Int. J. Numer. Methods Fluids 30, 977 (1999).

${ }^{72}$ T. H. Kuehn and R. J. Goldstein, "An experimental and theoretical study of natural convection in the annulus between horizontal concentric cylinders," J. Fluid Mech. 74, 695 (1976).

${ }^{73}$ Z. Chen, C. Shu, and D. Tan, "A simplified thermal lattice Boltzmann method without evolution of distribution functions," Int. J. Heat Mass Transfer 105, 741 (2017).

${ }^{74}$ I. Sezai and A. A. Mohamad, "Double diffusive convection in a cubic enclosure with opposing temperature and concentration gradients," Phys. Fluids 12, 2210 (2000).

${ }^{75}$ A. A. Mohamad, R. Bennacer, and J. Azaiez, "Double diffusion natural convection in a rectangular enclosure filled with binary fluid saturated porous media: The effect of lateral aspect ratio," Phys. Fluids 16, 184 (2004).

${ }^{76}$ P. J. Carreau, "Rheological equations from molecular network theories," Trans. Soc. Rheol. 16, 99 (1972).

${ }^{77}$ R. R. Huilgol, Fluid Mechanics of Viscoplasticity (Springer, 2015).

${ }^{78}$ R. R. Huilgol and G. H. R. Kefayati, "Natural convection problem in a Bingham fluid using the operator-splitting method," J. Non-Newtonian Fluid Mech. 220, 22 (2015).

${ }^{79}$ G. H. R. Kefayati and R. R. Huilgol, "Lattice Boltzmann method for simulation of mixed convection of a Bingham fluid in a lid-driven cavity," Int. J. Heat Mass Transfer 103, 725 (2016). 